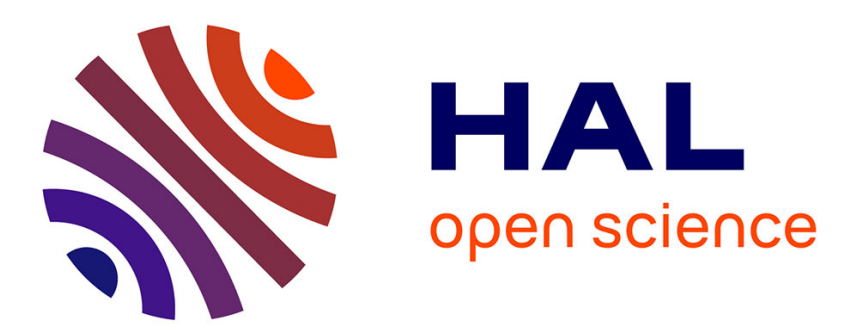

\title{
Separation and identification of structural modes in largely underdetermined scenarios using frequency banding
}

\author{
Roberto Castiglione, Jérôme Antoni, Luigi Garibaldi
}

\section{- To cite this version:}

Roberto Castiglione, Jérôme Antoni, Luigi Garibaldi. Separation and identification of structural modes in largely underdetermined scenarios using frequency banding. Journal of Sound and Vibration, 2018, 414, pp.192 - 217. 10.1016/j.jsv.2017.10.033 . hal-01714319

\author{
HAL Id: hal-01714319 \\ https://hal.science/hal-01714319
}

Submitted on 3 Sep 2021

HAL is a multi-disciplinary open access archive for the deposit and dissemination of scientific research documents, whether they are published or not. The documents may come from teaching and research institutions in France or abroad, or from public or private research centers.
L'archive ouverte pluridisciplinaire HAL, est destinée au dépôt et à la diffusion de documents scientifiques de niveau recherche, publiés ou non, émanant des établissements d'enseignement et de recherche français ou étrangers, des laboratoires publics ou privés. 


\title{
Separation and identification of structural modes in largely underdetermined scenarios using frequency banding
}

\author{
Roberto Castiglione ${ }^{\text {a, b }}$, Jerome Antoni ${ }^{\text {a, }}$, Luigi Garibaldi ${ }^{b}$ \\ a Laboratoire Vibrations Acoustique, Univ Lyon, INSA-Lyon, LVA EA677, F-69621, Villeurbanne, France \\ b Politecnico di Torino, Dipartimento di Ingegneria Meccanica e Aerospaziale, Corso Duca degli Abruzzi, 24, 10129 Torino, Italy
}

\section{A R T I C L E I N F O}

\section{Article history:}

Received 21 November 2016

Received in revised form 31 August 2017

Accepted 20 October 2017

\section{Keywords:}

Blind source separation

Operational modal analysis

System identification

Structural modes

Inverse problem

\begin{abstract}
A B S T R A C T
In recent years, blind source separation (BSS) has gained significant interest in the context of operational modal analysis, as a non-parametric alternative to the identification of mechanical structures from output-only measurements. One persisting limitation of most BSS methods, however, is to they cannot identify more active modes than the number of simultaneously measured outputs. The aim of this work is to propose a solution to the largely underdetermined case - where many more modes are to be identified than the number of available measurements - by dividing the frequency axis in subbands, such that each band provides an (over)determined problem where BSS can be applied separately. The approach comes with the proposal of a new second-order BSS that operates directly in the frequency domain and takes as an input the cross-spectral matrix of the data. A data augmentation technique is also devised to artificially increase the dimension of the measurements in severely undetermined scenarios. Finally, an identification algorithm is introduced that estimates the modal parameters of the separated structural modes. A remarkable aspect of these algorithms is that they are all based on the unified use of multifilters designed in the frequency domain, yet with different frequency bandwidths. Another particularity of the present paper is to demonstrate the validity of the proposed approach on several benchmark databases with various degrees of difficulty including complex modes, high modal overlap, singular modes, and the presence of engine harmonics. In all cases, the proposed methodology was efficient and, above all, easy to deal with even in largely undetermined cases.
\end{abstract}

(c) 2017 Elsevier Ltd. All rights reserved.

\section{Introduction}

The scope of operational modal analysis (OMA) is to identify the modal properties of a mechanical structure through the analysis of vibration data measured under operating conditions, when neither initial conditions nor artificial excitations are known. OMA is typically applied when it is complicated and/or expensive to deal with controlled vibration tests, for instance with very large structures (e.g. bridges, buildings) or when in situ excitation is hardly accessible (e.g. wind turbines). Many different techniques have been developed for OMA, from simple peak picking in the Fourier spectra to sophisticated system

\footnotetext{
* Corresponding author.

E-mail addresses: roberto.castiglione@polito.it (R. Castiglione), jerome.antoni@insa-lyon.fr (J. Antoni), luigi.garibaldi@polito.it (L. Garibaldi).
} 


\section{List of symbols ${ }^{1}$}

\section{ALS Alternative least squares \\ BSS Blind source separation \\ CP Complexity pursuit \\ CSM Cross-spectral matrix \\ DA Data augmentation \\ ESD Energy spectral density}

FD-BSS Frequency-domain blind source separation

GCP Generalized complexity pursuit

GSM Generalized spectral moment

ICA Independent component analysis

JAD Joint approximate diagonalization

MOF Modal overlap factor

OMA Operational modal analysis

PSD Power spectral density

PARAFAC Parallel factor analysis

RMS Root mean square value

SNR Signal-to-noise ratio

SOBI Second-order blind identification

SSI Stochastic subspace identification

M Number of channels

$N \quad$ Total number of active modes

$N_{i} \quad$ Total number of active modes in band $B_{i}$

$J \quad$ Number of FD-BSS filters in each band

$D \quad$ Number of GSM filters in each band

$A_{i} \quad$ Number of DA filters in band $B_{i}$

$B_{i} \quad i$-th frequency band used in FD-BSS

$\boldsymbol{y}(t) \quad$ Vector of measured structural responses (of dimension $M$ )

$\boldsymbol{n}(t) \quad$ Vector of additive noise (of dimension $M$ )

$\eta(t) \quad$ Vector of modal coordinates (of dimension $N$ )

$F_{S} \quad$ Sampling frequency (in $\left.\mathrm{Hz}\right)$

$T_{S} \quad$ Sampling period (in s)

$\Delta f \quad$ Frequency resolution of Fourier transform (in $\mathrm{Hz}$ )

$X(f) \quad$ Fourier transform of signal $\boldsymbol{x}(t)$

$\boldsymbol{Y}_{j}^{\mathrm{DA}}(f) \quad j$-th vector of spectra obtained by data augmentation

$\boldsymbol{Y}^{a u g}(f) \quad$ Complete vector of augmented data

$\mathbf{S}_{x x}(f) \quad$ Cross-spectral matrix of the signals in vector $\boldsymbol{x}(t)$

$\mathbf{S}_{x x, B_{i}}(f)$ Cross-spectral matrix of reduced dimension $N_{i}$

$\mathbf{S}_{x x, B_{i}}^{(j)} \quad$ Cross-spectral matrix weighted with $j$-th frequency gain and integrated in band $B_{i}$

$S_{\widehat{\eta}_{k}}(f) \quad$ Energy spectrum of $k$-th estimated modal coordinate

$\mathbf{S}_{y y}^{a u g}(f) \quad$ Cross-spectral matrix of augmented data

$\boldsymbol{\Phi} \quad$ Modal matrix

$\boldsymbol{\Phi}_{B_{i}} \quad$ Modal matrix of reduced dimension $N_{i}$

$\mathbf{A}^{+} \quad$ Pseudo-inverse of matrix $\mathbf{A}$

$\mathbf{A}^{T} \quad$ Transpose of matrix (or vector) $\mathbf{A}$

$\mathbf{A}^{H} \quad$ Transpose conjugate of matrix (or vector) $\mathbf{A}$

$G_{i j}^{\mathrm{BSS}}(f) \quad j$-th frequency gain of FD-BSS used in band $B_{i}$

$G_{i j}^{\mathrm{ID}}(f) \quad j$-th frequency gain of GSM used in band $B_{i}$

$G_{j}^{\mathrm{DA}}(f) \quad j$-th frequency gain of DA

$B_{k j}^{\mathrm{BSS}} \quad$ Bandwidth of $G_{i j}^{\mathrm{BSS}}(f)$ (in $\left.\mathrm{Hz}\right)$

$B_{k j}^{\mathrm{ID}} \quad$ Bandwidth of $G_{i j}^{\mathrm{ID}}(f)$ (in $\mathrm{Hz}$ )

$B_{j}^{\mathrm{DA}} \quad$ Bandwidth of $G_{j}^{\mathrm{DA}}(f)($ in $\mathrm{Hz}$ )

$f_{k} \quad$ Natural frequency of $k$-th mode (in $\mathrm{Hz}$ )

$\zeta_{k} \quad$ Damping ratio of $k$-th mode

$\lambda_{k} \quad$ Relaxation time of $k$-th mode (in $\mathrm{Hz}$ )

$\tilde{f}_{k} \quad$ Damped frequency of $k$-th mode (in $\mathrm{Hz}$ ) 
identification schemes (e.g. stochastic subspace identification (SSI)) [1,2,3]. Recently, blind source separation (BSS) has emerged as a valid non-parametric alternative in the scientific community for this purpose [6].

The objective of BSS is to extract from simultaneous measurements of the outputs of a system the respective contributions due to independent inputs, referred to as the "source signals" or the simply "sources" [4]. This is theoretically equivalent to decomposing a MIMO (multiple-input-multiple-output) system into a superposition of SIMO (single-input-multiple-output) systems. BSS operates from output measurements only, without requiring any prior knowledge on the system parameters, by exploiting some properties of independence of the sources. BSS has been applied in various fields involving communication, finance, astrophysics, bioengineering and more recently in structural dynamics for separating modal contributions from output-only measurements, which was found useful in OMA [6]. The advantage of BSS for OMA is that it is a non-parametric method. It thus offers an interesting alternative or a complement to parametric methods - such as SSI - which sometimes involve difficult model selection choices. In addition, once the modal contributions have been separated, modal parameters can be evaluated by using simple single-degree-of-freedom techniques, with much less effort than global identification approaches [5].

The first application of BSS in structural dynamics was reported in Ref. [7] which employed independent component analysis (ICA), an algorithm based on the use of fourth-order statistics of the data. It was closely followed by Refs. [8,9] where it was demonstrated that SOBI (second-order system identification), a second-order ${ }^{2}$ BSS algorithm based on the crosscorrelation of the data, improved the performance of separation as compared to ICA. From there on, several research works have compared BSS algorithms and investigated their limitations in OMA applications [10,11]. A theoretical analysis of second-order BSS is found in Ref. [12] which also proposed a solution that addresses the issue of complex mode shapes, heavily damped and strongly coupled modes. Another BSS criterion based on complexity pursuit (CP) was presented in Refs. [13-15], with marked potential. Reference [16] recently showed that CP is actually a second-order BSS algorithm quite similar to SOBI and proposed a generalization that improves its performance in terms of versatility and robustness. A comprehensive review of BSS in structural dynamics is provided in Ref. [6].

As pointed in Ref. [6], a persisting limitation of current BSS algorithms is that they can only separate a number of active modes equal to or less than the number of simultaneously measured responses, which is referred to as an (over)determined configuration. Specifically, if $M$ denotes the number of channels in the experiment (used in simultaneous recording) and $N$ the number of active modes, then conventional BSS algorithms require having $N \leq M$. This places a serious limitation in practice, which has been reported repeatedly in the literature. So far, this has mostly constricted the use of BSS to systems with few degrees of freedom or with a low modal density. A few BSS algorithms have been proposed in the literature to handle the underdetermined case (i.e. $N>M$ ), yet they seem limited to very restrictive scenarios where the number of active modes is only slightly greater than the number of channels (e.g. $N=M+1$ ). Hitherto, two main approaches seem to prevail: the first one involves some kind of tensor decomposition and the second one exploits the source sparsity. Methods involving tensor decomposition use multi-linear algebra in order to increase the dimension of the problem and make it overdetermined. They resort to techniques such as alternating least square (ALS) [17], canonical decomposition (CANDE-COMP) [18] and parallel factor analysis (PARAFAC) [19]. Applications of tensor decomposition in OMA are found in Refs. [20-23], yet they have had limited success thus far due to high computational costs and the difficulty to control convergence. The second approach exploiting source sparsity has proven more promising. Source sparsity means that there exists a domain of representation where the sources tend to occupy different regions of the space, with reduced overlap [24-27]. Source separation can thus be performed by confining the problem to subdomains where it locally becomes (over)determined [28]. With structural modes, sparsity is reached to some extent in the frequency domain where, at a given frequency, the number of significant overlapping modes is limited - this is opposite to the time domain, where at a given time all active modes in the system overlap. This generally holds provided that the modal overlap factor (MOF) (ratio between the 3-dB bandwidth and the average modal spacing) is small, i.e. in the so-called "modal" or "low-frequency" range where lightly damped and weakly coupled modes dominate. The idea was probably first demonstrated in Ref. [29] where BSS was solved with SOBI applied in frequency bands. Later, Refs. [30,32,33] independently investigated similar ideas, yet with somewhat more sophisticated methods. In the case of asynchronous free responses, sparsity was also be approached in the time-frequency domain [31].

This paper proceeds with the solution originally introduced in Ref. [29] because it is believed to be more amenable to handle largely underdetermined systems with many more modes than available measurements. This is achieved by decomposing the frequency range of interest into several subbands (possibly overlapping), so as to end up with a series of (over)determined BSS problems in each subband. Not only is this process straightforward and intuitive for the user - it requires no more than the inspection of the data spectra and the selection of bands by "dragging the mouse" - but it virtually has no limit in the number of modes that can be separated this way. However, splitting the problem into several bands in the frequency domain is found hardly compatible with state-of-the-art BSS algorithms which mostly operate in the time-domain. In Ref. [29], bandpass filters were apparently applied in the time domain as required by the use of SOBI, yet this is prone to produce spurious transient effects - thus placing a limit on the narrower filters - and it is numerically intensive because the cross-correlation matrix of the filtered signals has to be recomputed in each band.

\footnotetext{
1 Notational conventions:Bold lower case letters denote vectors, Bold upper case letters denote matrices, Statistical estimates are denoted with a hat symbol (e.g. $\widehat{\boldsymbol{\Phi}}$ for the estimate of $\boldsymbol{\Phi}$ ).

${ }^{2}$ A second-order BSS algorithm is one that uses only the second-order statistics of the data, e.g. the correlation functions or the cross-spectral matrix.
} 
A first objective of this paper is to introduce a frequency-domain algorithm - hereafter referred to as FD-BSS - that directly solves BSS in a given subband and in a computationally efficient way. The proposed algorithm is inspired by the frequencydomain version of the GCP algorithm (generalized CP) of Ref. [16] and thus pertains to second-order BSS methods. It takes as an input the cross-spectral matrix of the data, which has to be computed only once.

A second objective of the paper is to introduce a data augmentation (DA) technique that can be used in frequency bands when BSS still remains underdetermined (for instance in the presence of a very limited number of sensors) in order to recover an (over)determined configuration. Although not strictly necessary in general (in many cases, the banding strategy alone should be enough to turn the problem overdetermined), the proposed DA is found straightforward to apply in the frequency domain and thus it surely deserves attention.

A third objective is to introduce an identification algorithm to estimate the global modal parameters from the separated modal contributions in their frequency subbands. The approach makes use of generalized spectral moments (GSM) computed in subbands and eventually requires solving a linear system of equations. As far as the authors know, this identification algorithm is original in the OMA landscape. Besides its simplicity, it is has also been found to enjoy very good performance.

Finally, a last objective of the paper is to demonstrate the feasibility of the methodology on experimental data with various degrees of difficulty, including instances with complex modes, high modal overlap, singular modes, and the presence of engine harmonics. Consequently, several experimental cases are reported in order to highlight the potential and versatility of the proposed algorithms.

As a final remark, it is noteworthy that the three objects of the paper, FD-BSS, DA and GSM-based identification are all rooted on the definition of multi-filters. This provides to the proposed methodology an unexpected unifying concept.

This paper is organized as follows. Section 2 first recalls the basics on BSS and then introduces the FD-BSS algorithm. Next, section 3 addresses the identification of modal parameters with GSM. Section 4 deals with the DA strategy. Section 5 then resumes the main findings in a complete methodology and provides guidelines to the user as how to apply it. Finally, the focus of the work switches to applications on several numerical and experimental data.

\section{Blind source separation in the frequency domain (FD-BSS)}

The modal expansion theorem expresses any configuration of a multi degree-of-freedom system as a linear combination of its eigenvectors. This is possible because eigenvectors are linearly independent and form a basis in the system configuration space. Let vector $\boldsymbol{y}(t) \in \mathbb{C}^{M}$ contain the $M$ measured structural responses of a system with $N$ active modes (in a frequency band of interest), vector $\boldsymbol{\eta}(t) \in \mathbb{C}^{N}$ denote the modal coordinates, $\boldsymbol{\Phi} \in \mathbb{C}^{M \times N}$ be the modal matrix whose columns are filled by the mode shapes, and vector $\boldsymbol{n}(t) \in \mathbb{C}^{M}$ contain possible measurement noise (complex-valued data are considered for the sake of generality); the modal expansion in matrix form then reads

$$
\boldsymbol{y}(t)=\boldsymbol{\Phi} \boldsymbol{\eta}(t)+\boldsymbol{n}(t) .
$$

This formulation happens to be similar to the BSS model, where the modal coordinates and modal matrix can be seen, respectively, as the "sources" and the "mixing matrix". ${ }^{3}$ Consequently, it has been recognized that BSS can be applied to structural data measured in operational condition - i.e. without the knowledge of the excitations - in order to recover modal parameters, given only the assumption of mutual independence of the modal coordinates [6]. Mathematically, the problem is solved by "blindly" determining an estimate of the modal matrix, say $\widehat{\boldsymbol{\Phi}}$ - which provides the mode shapes - and its pseudoinverse $\widehat{\boldsymbol{\Phi}}^{+}$from which the modal coordinates can be estimated as

$$
\widehat{\boldsymbol{\eta}}(t)=\widehat{\boldsymbol{\Phi}}^{+} \boldsymbol{y}(t)
$$

provided that $M \geq N$. How to find the modal matrix $\widehat{\boldsymbol{\Phi}}$ or its inverse from the measurement of the output data $\boldsymbol{y}(t)$ only is the aim of BSS (readers are invited to refer to Ref. [1] for a general introduction to BSS and to the review paper [6] for its application to the separation of structural modes).

As explained in the introduction, one of the challenges of current BSS algorithms is to separate more structural modes than the number of available channels, i.e. to solve the undetermined configuration $M<N$. Although several BSS methods have been investigated in the literature, an algorithm inspired by the recently proposed GCP method is promoted in this paper because of its many advantages [16]. First, it generalizes other methods which currently serve as baselines in the state-of-theart in OMA, such as SOBI and CP. Second, it is quite flexible to apply as it comes with a numerically efficient algorithm that is transparent to the end-user. Third, it is easily applicable in the frequency domain, in particular in subbands, which is the approach advocated in this paper to tackle the undetermined case. It is noted that other approaches than GCP may be taken to arrive at a similar frequency-domain algorithm. The derivation of the FD-BSS algorithm given hereafter can actually be read independently of the material of Ref. [16].

\footnotetext{
${ }^{3}$ The usual notation in BSS is in the form $\boldsymbol{y}(t)=\mathbf{A s}(t)+\boldsymbol{n}(t)$ (so-called "instantaneous mixture") where $\boldsymbol{s}(t)$ is the vector of source signals and $\boldsymbol{A}$ the mixing matrix; the aim of BSS is to recover the sources $\boldsymbol{s}(t)$ from observing $\boldsymbol{y}(t)$ only, without the knowledge of the mixing matrix $\boldsymbol{A}$ [2].
} 
Let $\boldsymbol{Y}(f) \in \mathbb{C}^{M}$ denote the Fourier transform of $\boldsymbol{y}(t) \in \mathbb{C}^{M}$. Next, let us define the cross-spectral matrix (CSM)

$$
\mathbf{S}_{y y}(f) \doteq \boldsymbol{Y}(f) \boldsymbol{Y}(f)^{H}
$$

(superscript ${ }^{H}$ stands for the complex-conjugate operator) where no averaging is considered at this place. Using Eq. (1), it follows that

$$
\mathbf{S}_{y y}(f)=\boldsymbol{\Phi} \mathbf{S}_{\eta \eta}(f) \boldsymbol{\Phi}^{H}+\mathbf{S}_{n n}(f)
$$

where $\mathbf{S}_{\eta \eta}(f) \in \mathbb{C}^{M}$ and $\mathbf{S}_{n n}(f) \in \mathbb{C}^{M \times M}$ stand for the CSMs of the modal coordinates and additive noise, respectively. Let us now consider a frequency band $B_{i}=\left[f_{L}^{i}, f_{U}^{i}\right]$, defined by its lower and upper frequencies, $f_{L}^{i}$ and $f_{U}^{i}$, respectively, that includes a few, say $N_{i}$, modes of interest. Equation (4) then becomes

$$
\mathbf{S}_{y y}(f)=\boldsymbol{\Phi}_{B_{i}} \mathbf{S}_{\eta \eta, B_{i}}(f) \boldsymbol{\Phi}_{B_{i}}^{H}+\mathbf{S}_{n n}(f), \quad f \in B_{i}
$$

where matrix $\boldsymbol{\Phi}_{B_{i}} \in \mathbb{C}^{M \times N_{i}}$ contains the mode shapes in band $B_{i}$ only and $\mathbf{S}_{\eta \eta, B_{i}}(f) \in \mathbb{C}^{N_{i} \times N_{i}}$ is the corresponding CSM of modal coordinates. A strict equality is kept in Eq. (5) because the effect of out-of-band modes in band $B_{i}$ - even though assumed with a negligible contribution - can always be reassigned to the noise CSM $\mathbf{S}_{n n}(f)$. Since the dimension of the mixing matrix $\boldsymbol{\Phi}_{B_{i}}$ is reduced, the problem is now more easily solved by BSS. In particular, the undetermined case $M<N$ is converted into an (over) determined case when $N_{i} \leq M$. This situation will be considered from now on, while section 4 will address the more general case where Eq. (5) is possibly still undetermined.

The first step of the FD-BSS algorithm is to transform the functional equation (5) into an algebraic equation by integrating the CSM over the frequency variable. Namely, let us define

$$
\mathbf{S}_{x x, B_{i}}^{(1)} \doteq \int_{f_{L}^{i}}^{f_{U}^{i}} \mathbf{S}_{x x}(f) d f, \quad x \in\{y, \eta, n\}
$$

Thus, Eq. (5) becomes

$$
\mathbf{S}_{y y, B_{i}}^{(1)}=\boldsymbol{\Phi}_{B_{i}}\left\lceil\mathbf{S}_{\eta \eta, B_{i}}^{(1)}\right\rfloor \boldsymbol{\Phi}_{B_{i}}^{H}+\mathbf{S}_{n n, B_{i}}^{(1)} .
$$

It is noteworthy that $\left[\mathbf{S}_{\eta \eta, B_{i}}^{(1)}\right\rfloor$ is well approximated by a diagonal matrix due to the fact that modal coordinates are nearly uncorrelated [5], a property which is emphasized by the notation $\lceil\cdots\rfloor$. Similarly, the CSM of noise might be reasonably assumed with a dominant diagonal, although the presence of out-of-band modes will occupy off-diagonal entries. Therefore, Eq. (7) gives good hope that $\boldsymbol{\Phi}_{B_{i}}$ can be recovered from the diagonalization of matrix $\mathbf{S}_{y y, B_{i}}^{(1)}$. However, it is well known in BSS that a simple eigenvalue decomposition (e.g. as would be performed with Principal Component Analysis) is not enough and that at least another equation is necessary to recover the mixing matrix uniquely. This is the second step of FD-BSS, which repeats the same integration process leading to Eq. (7) after pre-multiplying the CSM $\mathbf{S}_{y y}(f)$ in band $B_{i}$ with the frequency gains of a family of arbitrary but linearly independent filters, say $G_{i j}^{\mathrm{BSS}}(f)$, indexed by $j=1, \ldots, J, J \geq 2$. Let us define

$$
\mathbf{S}_{x x, B_{i}}^{(j)} \doteq \int_{f_{L}^{i}}^{f_{U}^{i}} \mathbf{S}_{x x}(f) G_{i j}^{\mathrm{BSS}}(f) d f, \quad x \in\{y, \eta, n\}
$$

with the convention $G_{i 1}^{\mathrm{BSS}}(f)=1$. Therefore, Eq. (5) becomes

$$
\mathbf{S}_{y y, B_{i}}^{(j)}=\boldsymbol{\Phi}_{B_{i}}\left[\mathbf{S}_{\eta \eta, B_{i}}^{(j)} \mid \boldsymbol{\Phi}_{B_{i}}^{H}+\mathbf{S}_{n n, B_{i}}^{(j)}, \quad j=1, \ldots, J\right.
$$

where matrices $\mathbf{S}_{\eta \eta, B_{i}}^{(j)}$ and $\mathbf{S}_{n n, B_{i}}^{(j)}$ are well approximated by diagonal forms for the same reasons as before. In addition, if the noise has a broadband spectrum and the frequency gains are chosen such that $\int_{f_{L}^{i}}^{f_{U}^{i}} G_{i j}^{\mathrm{BSS}}(f) d f=0$, it comes that $\mathbf{S}_{n n, B_{i}}^{(j)} \approx 0$. Equation (9) now provides a system of $J$ equalities to be solved for $\boldsymbol{\Phi}_{B_{i}}$. Because of the presence of additive noise and small contributions from out-of-band modes, this is solved in FD-BSS by searching for an estimate of $\boldsymbol{\Phi}_{B_{i}}$ that makes the matrices in set $\left\{\mathbf{S}_{y y, B_{i}}^{(j)} ; j=1, \ldots, J\right\}$ as diagonal as possible in the least square sense, i.e. 


$$
\widehat{\boldsymbol{\Phi}}_{B_{i}}=\operatorname{Argmin} \sum_{j=1}^{J}\left\|\operatorname{Off}\left\{\mathbf{S}_{y y, B_{i}}^{(j)}-\mathbf{\Phi}_{B_{i}} \mathbf{S}_{\eta \eta, B_{i}}^{(j)} \boldsymbol{\Phi}_{B_{i}}^{H}\right\}\right\|^{2}, J \geq 2
$$

where $\|\cdots\|$ stands for the Frobenius norm and Off for the operator that zeroes the diagonal elements of a matrix. The minimization in Eq. (10) is performed by using the joint approximate diagonalization (JAD) algorithm [37]. Once an estimate $\widehat{\boldsymbol{\Phi}}_{B_{i}}$ is found from JAD, the last step of FD-BSS is to recover the mode shapes in band $B_{i}$ as the columns of $\widehat{\boldsymbol{\Phi}}_{B_{i}}$ and the corresponding modal coordinate spectra, $\widehat{\eta}_{B_{i}}(f) \in \mathbb{C}^{N_{i}}$, from the pseudo inversion

$$
\widehat{\boldsymbol{\eta}}_{B_{i}}(f)=\widehat{\boldsymbol{\Phi}}_{B_{i}}^{+} \boldsymbol{Y}(f)=\left(\widehat{\boldsymbol{\Phi}}_{B_{i}}^{H} \widehat{\boldsymbol{\Phi}}_{B_{i}}\right)^{-1} \widehat{\boldsymbol{\Phi}}_{B_{i}}^{H} \boldsymbol{Y}(f), f \in B_{i}
$$

Note that the estimated modal spectra are valid in band $B_{i}$ only, yet they contain information for identifying the global modal parameters as demonstrated in the next section. The FD-BSS algorithm is summarized in Table 1. A Matlab code is made available at the following address: http://www.mathworks.com/matlabcentral/fileexchange/.

Similar to GCP [16], the proposed FD-BSS algorithm can be applied with a variety of different multi-filters, which makes it rather general. As a particular case, $\mathrm{CP}$ is recovered with two high-pass filters $(J=2)$ and SOBI multiple delay filters [16]. As discussed in section 5, a convenient choice is to design the multi-filters with sinusoidal frequency gains in band $B_{i}$ with a number of oscillations increasing with index $j$.

It should be reminded here that a fundamental assumption beyond the FD-BSS algorithm - also shared by most other BSS algorithms - is that the modal contributions are nearly uncorrelated -i.e. their CSM is diagonal. In theory, this condition should be satisfied for structural mode with negligible modal overlap, yet the FD-BSS algorithm has been found remarkably robust against this assumption. This will be verified in the examples of section 6 , where MOFs greater than $100 \%$ will be dealt with.

\section{Identification of modal parameters with generalized spectral moments (GSM)}

Once the modal contributions have been separated by BSS, the problem is put in a simplified form for identifying modal parameters since it involves only responses to single-degree-of-freedom systems. Whereas the columns of the modal matrix $\boldsymbol{\Phi}$ are returned as a natural output of the FD-BSS algorithm, the identification of the global modal parameters requires further processing. Local non-parametric methods such as the $3 \mathrm{~dB}$ bandwidth or the logarithmic decrement are often used, yet they are subjected to a certain degree of approximation. In particular, the $3 \mathrm{~dB}$ bandwidth method is rather sensitive to the presence of residual noise whereas the logarithmic decrement is difficult to apply in a narrow band. A parametric identification procedure is proposed in this section, which is perfectly in line with the multi-filter frequency-domain approach of the FD-BSS algorithm. It takes explicit account of residual noise and operates in frequency bands. The solution is rooted on generalized spectral moments (GSM) and has been found to a have performance nearly identical to the maximum likelihood solution - which has asymptotically the smallest variance - yet with reduced complexity; therefore, only the former is reported here. As far as the authors know, the proposed method is new and given here for the first time.

To start with, let us consider the Fourier transform of the $k$-th extracted modal contribution, $\widehat{\eta}_{k}(f)$, as returned by Eq. (11), in a frequency band $B_{k}^{I D}$ (superscript ID stands for "IDentification") that includes the mode frequency (from now on index $k \in\{1, \ldots, N\}$ will relate to the mode number in the initial vector $\left.\boldsymbol{\eta}(t) \in \mathbb{C}^{N}\right)$. By definition, this is the response to a singledegree-of-freedom system. Assuming viscous damping (other damping models can be considered at this stage, e.g. structural damping, without changing the principle of the method), this is expressed in the frequency domain as

$$
\widehat{\eta}_{k}(f)(2 \pi)^{2}\left(-f^{2}+f_{k}^{2}+2 \mathrm{j} \zeta_{k} f f_{k}\right)=F_{k}(f)(2 \pi f \mathrm{j})^{d}, f \in B_{k}^{I D}
$$

where $\zeta_{k}$ and $f_{k}$ stand for the damping ratio and the natural frequency, respectively, of the $k$-th mode, $d=0,1,2$ whether displacement, velocity, or acceleration is measured, $F_{k}(f)$ stands for an unknown white excitation and $\mathrm{j}$ is the imaginary number. The aim is to estimate $\zeta_{k}$ and $f_{k}$ from the observation of $\widehat{\eta}_{k}(f)$ only. Since the phase of the excitation is unknown, it makes sense to consider only the squared magnitude of Eq. (12). Introducing the spectrum $S_{\widehat{\eta}_{k}}(f)=\left|\widehat{\eta}_{k}(f)\right|^{2}$ of the separated

Table 1

The FD-BSS algorithm.

1) Compute the CMS $\boldsymbol{S}_{y y}(f)$ according to Eq. (3).

2) Compute the integrated CSMs $\mathbf{S}_{y y, B_{i}}^{(j)}$ in band $B_{i}$ based on the frequency gain $G_{i j}^{\mathrm{BSS}}(f)$ for $j=1, \ldots J$, according to Eq. (8).

3) Find an estimate $\widehat{\boldsymbol{\Phi}}_{B_{i}}$ of the matrix that contains the mode shapes in band $B_{i}$ by jointly diagonalizing the CSMs $\mathbf{S}_{y y, B_{i}}^{(j)}$ in the least square sense according

to Eq. (10).

4) Recover the modal coordinates in band $B_{i}$ by applying the pseudo inverse $\widehat{\boldsymbol{\Phi}}_{B_{i}}^{+}$to the measured structural responses according to Eq. (11). 
mode - which is directly obtained as one of the diagonal elements of $\widehat{\boldsymbol{\Phi}}_{B_{i}}^{+} \mathbf{S}_{y y}(f) \widehat{\boldsymbol{\Phi}}_{B_{i}}^{+H}$ (see Eq. (11)) - this yields

$$
S_{\widehat{\eta}_{k}}(f) \frac{(2 \pi)^{4}}{(2 \pi f)^{2 d}}\left(f^{4}+f_{k}^{4}+4 \zeta_{k}^{2} f^{2} f_{k}^{2}-2 f^{2} f_{k}^{2}\right)=\left|F_{k}(f)\right|^{2}
$$

This equation could be solved for $\zeta_{k}$ and $f_{k}$ if the energy spectrum $\left|F_{k}(f)\right|^{2}$ was known. Although the spectrum is not measured, it is constant on the average because of the whiteness assumption of OMA (at least in the band of interest). Therefore, if $G^{\mathrm{ID}}(f)$ denotes a zero mean function in band $B_{k}^{\mathrm{ID}}$ such that

$$
\int_{B_{k}^{\mathrm{ID}}} G^{\mathrm{ID}}(f) d f=0
$$

then $\int_{B_{k}^{\mathrm{ID}}}\left|F_{k}(f)\right|^{2} G^{\mathrm{ID}}(f) d f=0$ will hold on the average. Let us now introduce a set of $D$ such functions, $\left\{G_{k j}^{\mathrm{ID}}(f) ; j=1, \ldots, D\right\}$, linearly independent in band $B_{k}^{\mathrm{ID}}$, with the convention $G_{k 1}^{\mathrm{ID}}(f)=1$. Next, let us define the $p$-th order GSM of the $k$-th extracted modal contribution as

$$
m_{k, j, p} \doteq(2 \pi)^{2(2-p)} \int_{B_{k}^{1 \mathrm{D}}} G_{k j}^{\mathrm{ID}}(f)(f) S_{\widehat{\eta}_{k}}(f)(2 \pi f)^{2(p-d)} d f .
$$

Multiplying each term in Eq. (13) by $G_{k j}^{\mathrm{ID}}(f)$ and integrating in band $B_{k}^{\mathrm{ID}}$, it then comes

$$
m_{k, j, 2}+a \cdot m_{k, j, 1}+b \cdot m_{k, j, 0}=c \cdot \delta_{j, 1}, \quad j=1, \ldots, D
$$

where $\delta_{j, 1}=1$ if $j=1$ and 0 otherwise, $a=2 f_{k}^{2}\left(2 \zeta_{k}^{2}-1\right), b=f_{k}^{4}$ and $c=\int_{B_{k}^{\mathrm{DI}}}\left|F_{k}(f)\right|^{2} d f$. The system of $D$ equation (16) is linear in the 3 unknowns $a, b$ and $c$ and is thus solved without difficulty with $D \geq 3$. For instance, by writing down the equations up to $j=3$, it comes

$$
\left[\begin{array}{ccc}
m_{k, 1,1} & m_{k, 1,0} & -1 \\
m_{k, 2,1} & m_{k, 2,0} & 0 \\
m_{k, 3,1} & m_{k, 3,0} & 0 \\
\vdots & \vdots & \vdots
\end{array}\right]\left[\begin{array}{c}
a \\
b \\
c
\end{array}\right]=\left[\begin{array}{c}
-m_{k, 1,2} \\
-m_{k, 2,2} \\
-m_{k, 3,2} \\
\vdots
\end{array}\right]
$$

which is easily inverted. Finally, the modal parameters are estimated as

$$
\begin{aligned}
f_{k} & =\sqrt[4]{b} \\
\zeta_{k} & =\sqrt{\frac{1}{2}\left(1+\frac{a}{2 \sqrt{b}}\right)} .
\end{aligned}
$$

In practice, it will be again convenient to design the frequency gains $G_{k j}^{\mathrm{ID}}(f)$ as sinusoidal functions with increasing number of oscillations with index $j$ - while still satisfying condition (14) in band $B_{k}^{\mathrm{ID}}$. The authors have found excellent results using this design.

Eventually, the global modal parameters found by the spectral moment method may be used to initialize more sophisticated identification algorithms, such as the maximum likelihood method. However, it has been found that, in the present context, estimates based on GSMs have nearly similar performance, while being less complex. This is the reason why the latters only will be used in the experimental cases of section 6 .

\section{Data augmentation by filtration (DA)}

Situations may still arise where the number of active modes in a frequency band - whatever narrow it is - remains greater than the number of channels. With the notations of section 3, this corresponds to the underdetermined situation $N_{i}<M$. For instance this would be the case with a very limited number of channels and a high modal density. A solution is introduced here - inspired from the trick used in SSI [38], yet reconsidered in the frequency-domain - which can fix the problem based on DA. It happens to fit very well in the multi-filter frequency-domain approach that has been followed so far. 
For simplicity, let us first consider the case of noise-free transient data. The measurement is thus the vector of impulse responses

$$
\boldsymbol{h}(t)=H(t) \sum_{k=1}^{N} \phi_{k} e^{-\lambda_{k} t} e^{\mathrm{j} 2 \pi \tilde{f}_{k} t}
$$

where $\lambda_{k}$ and $\tilde{f}_{k}$ stand for the relaxation time and the damped frequency (the tilde symbol is used to avoid confusion with the natural frequency), respectively, of the $k$-th mode, $\phi_{k}$ stands for the $k$-th mode shape (i.e. the $k$-th column of matrix $\boldsymbol{\Phi}$ ) and where $H(t)$ stands for the Heaviside function (unit modal amplitudes are assumed without loss of generality). Let us now consider the same data shifted by $p$ time samples and cut at negative times,

$$
H(t) \boldsymbol{h}\left(t+p T_{s}\right)=H(t) \sum_{k=1}^{M} \boldsymbol{\phi}_{k} e^{-\lambda_{k}\left(t+p T_{s}\right)} e^{\mathrm{j} 2 \pi \tilde{f}_{k}\left(t+p T_{s}\right)}=H(t) \sum_{k=1}^{M} \boldsymbol{\phi}_{k} \beta_{k}^{p} e^{-\lambda_{k} t} e^{\mathrm{j} 2 \pi \tilde{f}_{k} t},
$$

with $T_{s}$ the sampling period and $\beta_{k}^{p}=\left(e^{-\lambda_{k} T_{s}+\mathrm{j} 2 \pi \tilde{f}_{k} T_{s}}\right)^{p}$. This is seen to produce a vector of impulse responses with the same global modal parameters $\left(\lambda_{k}\right.$ and $\left.\tilde{f}_{k}\right)$ and same modes shapes $\phi_{k}$ as in Eq. (19), yet with different modal amplitudes. Shifted data can thus be seen as new measurements because they are obtained as a linear combination of the system modes with different coefficients. Similar results are eventually obtained by considering any linear combination of shifted data, $H(t) \sum_{p} g_{p} \boldsymbol{h}\left(t+p T_{s}\right)$, where the set of coefficients $g_{p}$ defines a linear filter. Next, proceeding with forced responses to a white noise excitation - (whiteness is the fundamental assumption behind all OMA methods) it can be shown that the same procedure applies to the cross-correlation matrix of the data.

An approximation of this transformation is easily obtained from the Fourier transform $\boldsymbol{Y}(f)$ used in the FD-BSS algorithm of section 2. Denoting $G_{j}^{\mathrm{DA}}(f), j=1, \ldots, A$, the frequency gain (Fourier transform of a set $j$ of coefficients $g_{p j}$ ), a set of $D$ new data its thus generated as

$$
\boldsymbol{Y}_{j}^{\mathrm{DA}}(f)=\boldsymbol{Y}(f) G_{j}^{\mathrm{DA}}(f), \quad j=1, \ldots, A
$$

Equation (21) comes as an approximation of Eq. (20) since it does not include the effect of the Heaviside function present in Eq. (20), which would correspond to a further convolution of $\boldsymbol{Y}(f) G_{j}^{\mathrm{DA}}(f)$ with $\frac{1}{2} \delta(f)+(\mathrm{j} 2 \pi f)^{-1}$, the Fourier transform of $H(t)$. As demonstrated in the experimental results of section 4, this approximation has been found good enough in practice.

The frequency gains used in transformation (21) share some similarity with the frequency gains used in FD-BSS. Indeed, they can be designed with the same structure, i.e. with a sinusoidal shape and increasing numbers of oscillations with index $j$. However, one patent difference is that they apply to the full frequency band, whereas the FD-BSS filters apply in narrow bands. Therefore, there is only one set of "macro" filters used for DA (in full band), whereas there are several sets of "micro" filters used for FD-BSS (in several subbands).

Finally, it is noteworthy that DA can be directly applied to the CSM of the structural responses. Let $\boldsymbol{Y}^{\text {aug }}(f)=\left[\begin{array}{llll}\boldsymbol{Y}(f)^{T} & \boldsymbol{Y}_{1}^{\mathrm{DA}}(f)^{T} & \cdots & \boldsymbol{Y}_{A}^{\mathrm{DA}}(f)^{T}\end{array}\right]^{T} \in \mathbb{C}^{M(A+1)}$ denote the augmented of Fourier transforms; then, from Eq. (21), it is

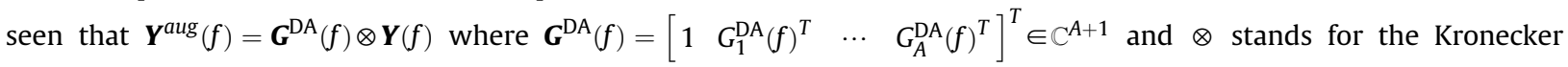
product. Therefore, the augmented CSM, say $\mathbf{S}_{y y}^{a u g}(f) \in \mathbb{C}^{M(A+1) \times M(A+1)}$, is

$$
\mathbf{S}_{y y}^{a u g}(f) \doteq \boldsymbol{Y}^{a u g}(f) \boldsymbol{Y}^{a u g}(f)^{H}=\left(\boldsymbol{G}^{\mathrm{DA}}(f) \boldsymbol{G}^{\mathrm{DA}}(f)^{H}\right) \otimes \mathbf{S}_{y y}(f)
$$

\section{Methodological progress}

This section collects the results of the previous sections to arrive at a complete methodology. It discusses the design of multi-filters, which is a common thread of the present frequency-domain approach and it eventually provides guidelines for the division of the frequency axis in subbands, which ends up being the only demand for the final user's intervention.

\subsection{A full frequency-domain process}

The proposed methodology to separate structural modes in the undetermined case is rooted on the three algorithms of sections 2, 3 and 4. The first one is a new BSS solution, FD-BSS, that operates in frequency subbands and returns the mode shapes together with the corresponding modal contributions. The second one is a parametric identification method based on GSMs that returns estimates of the damping ratios and natural frequencies of the separated modes. The third algorithm 
Table 2

Complete methodology to separate structural modes in the (largely) undetermined case.

1) Divide the frequency axis into (possibly overlapping) frequency bands $B_{i}, i=1, \ldots, B$.

In each band $B_{i}$

2) Resort to DA when the number of modes $N_{i}$ is still greater than the number of channels $M$.

3) Apply FD-BSS to recover the mode shapes and separate the modal contributions.

4) Apply GSM-based identification on the separated modal contributions to estimate the global modal parameters.

artificially augments the number of channels when the problem remains undetermined in a frequency band. The complete methodology is summarized in Table 2 and also illustrated in Fig. 1.

It is remarkable that the full process requires only frequency data as its input, in the form of the CSM, independently of the statistical properties of the original time signals. This implies that it applies equally well to transient or stationary random signals, or a combination of both. For the same reason, it also comes with a potential huge reduction of the data storage requirement. Although the (non-parametric) frequency domain approach if often criticized in modal analysis because it suffers from leakage errors [39], it must highlighted here that the latter do not interfere with the FD-BSS algorithm since Eq. (5) continues to hold true even in their presence. The GSM-based identification is also found very robust in this respect since leakage is averaged off in the computation of GSMs. Consequently, the proposed methodology hardly depends on how the CSM is computed (e.g. type of data window, type of spectral estimator).

\subsection{Design of frequency gains}

The FD-BSS, the GSM-based identification and the DA algorithms are all rooted on the use of multi-filters. As detailed in sections 2,3 and 4 , a set of frequency gains $\left\{G_{k j}^{\mathrm{BSS}}(f)\right\}$ is used for mode separation, a set $\left\{G_{j}^{\mathrm{DA}}(f)\right\}$ for DA and a set $\left\{G_{k j}^{\mathrm{ID}}(f)\right\}$ for modal parameter identification. Although these three sets are generally different, they are strongly dependent and may involve similar filter designs.

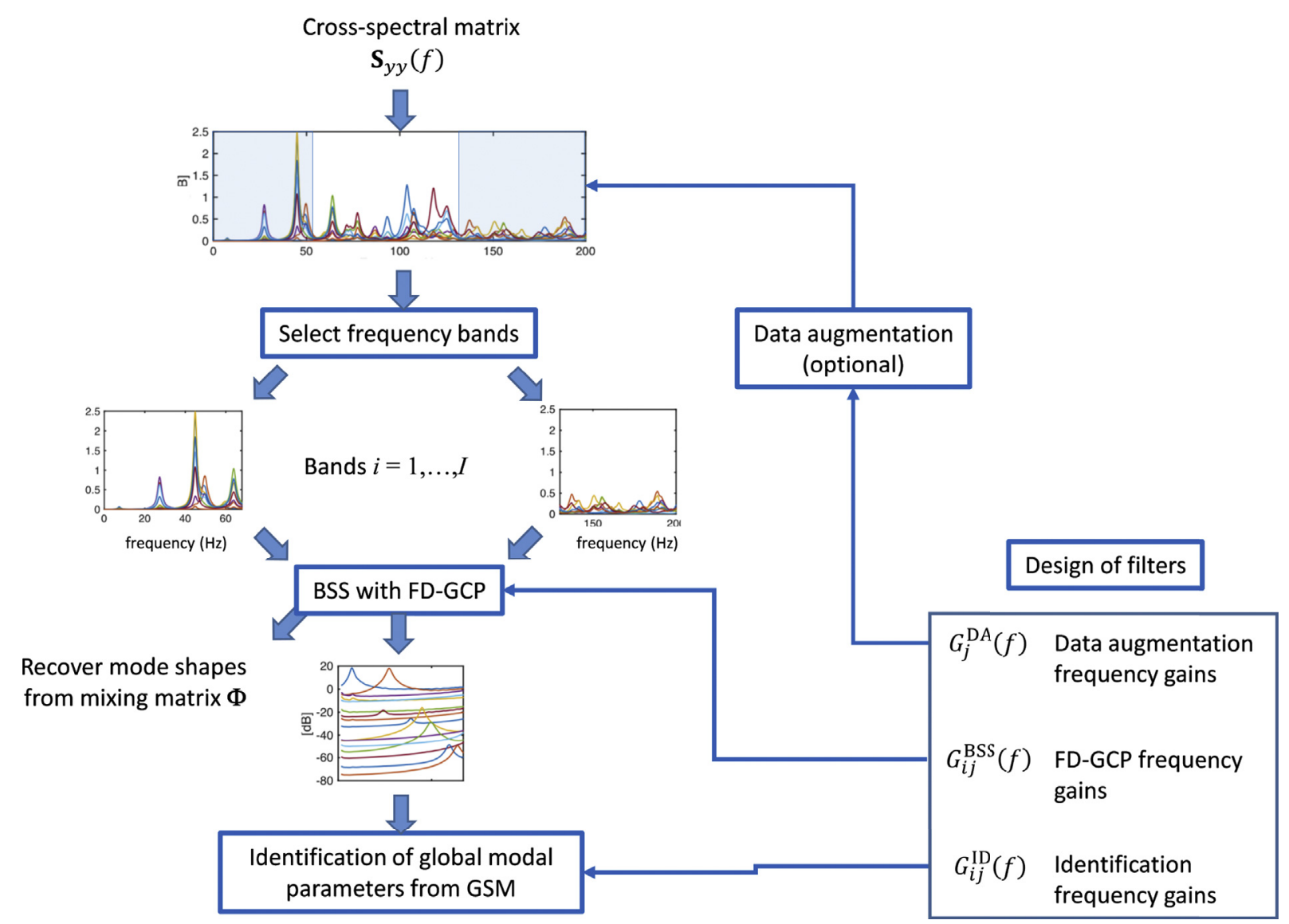

Fig. 1. Flowchart of the proposed methodology. 
a) Support sets

As mentioned in section 4, the frequency gains of DA should span the full frequency range that contains all the modes of interest and should therefore cover the frequency gains of FD-BSS. Besides, the frequency gains of FD-BSS should cover those of GSMs since modal identification applies to modal contributions whose separation is valid only in the bands of the FD-BSS algorithm (see Fig. 1). Therefore, the support sets of the frequency gains are actually interlaced such that

$$
\forall\left(j_{1}, j_{2}, j_{3}\right), \operatorname{supp}\left(G_{k j_{1}}^{\mathrm{ID}}\right) \subseteq \operatorname{supp}\left(G_{k j_{2}}^{\mathrm{BSS}}\right) \subseteq \operatorname{supp}\left(G_{j_{3}}^{\mathrm{DA}}\right)
$$

where $\operatorname{supp}\left(G_{\ldots}^{\mathrm{X}}\right)$ denotes the frequency support (i.e. the interval where the gain is non-zero) of $G_{\ldots}^{\mathrm{X}}(f), X \in\{I D, B S S, D A\}$. This also implies that the corresponding bandwidths (i.e. the lengths of the supports) must satisfy

$$
\forall\left(j_{1}, j_{2}, j_{3}\right), \Delta f<B_{k j_{1}}^{\mathrm{ID}} \leq B_{k j_{2}}^{\mathrm{BSS}} \leq B_{j_{3}}^{\mathrm{DA}} \leq \frac{1}{2} F_{S}
$$

where $F_{S}=1 / T_{S}$ stands for the sampling frequency and $\Delta f$ for the frequency resolution of the CSM. The first inequality reflects the constraint that a frequency band used in FD-BSS cannot be smaller than the available frequency resolution. These conditions are actually less stringent to apply than it appears, since in the three sets of frequency gains only the support of $\left\{G_{k j}^{\mathrm{BSS}}(f)\right\}$ is crucial - this is the subject of the next subsection. The settings of the other supports directly result from the latter one; reasonable default choices are $\operatorname{supp}\left(G_{j_{3}}^{\mathrm{DA}}\right)=\left[0 ; \frac{1}{2} F_{S}\right]$ and $\operatorname{supp}\left(G_{k j_{1}}^{\mathrm{ID}}\right)$ equal to the intersection of $\operatorname{supp}\left(G_{k j_{2}}^{\mathrm{BSS}}\right)$ and the $3 d B$-bandwidth interval of $\widehat{\eta}_{k}(f)$. These settings can be set automatically and have been used in the experimental cases of section 6.

b) Filter architecture

As previously mentioned, several designs are conceivable for the frequency gains used in FD-BSS, DA and GSM. According to the authors' experience, excellent results have been obtained by using smooth oscillating functions with increasing number of periods. Hence, for the sake of simplicity, all frequency gains have been designed with the same sinusoidal shape, given hereafter for $G_{i j}^{\mathrm{BSS}}(f)$ (similar formulas hold for $G_{i j}^{\mathrm{ID}}(f)$ and $G_{j}^{\mathrm{DA}}(f)$ after modifying the support sets):

$$
G_{i j}^{\mathrm{BSS}}(f)=\left\{\begin{array}{l}
\cos \left(\pi\left(f-f_{L}^{i}\right) j /\left(f_{U}^{i}-f_{L}^{i}\right)\right), j \text { odd } \\
\sin \left(\pi\left(f-f_{L}^{i}\right) j /\left(f_{U}^{i}-f_{L}^{i}\right)\right), j \text { even }
\end{array}, \quad f_{L}^{i} \leq f \leq f_{U}^{i} .\right.
$$

This is illustrated in Fig. 2.

The number J of FD-BSS filters must be taken larger than or equal to 2 for the optimization problem in Eq. (10) to find an unique solution. It has been found that the proposed FD-BSS algorithm has low sensitivity to the value of J, yet larger values will help in the presence of noise - this is reminiscent to the behavior of SOBI, as studied in Ref. [34]. Based on Eq. (25), the

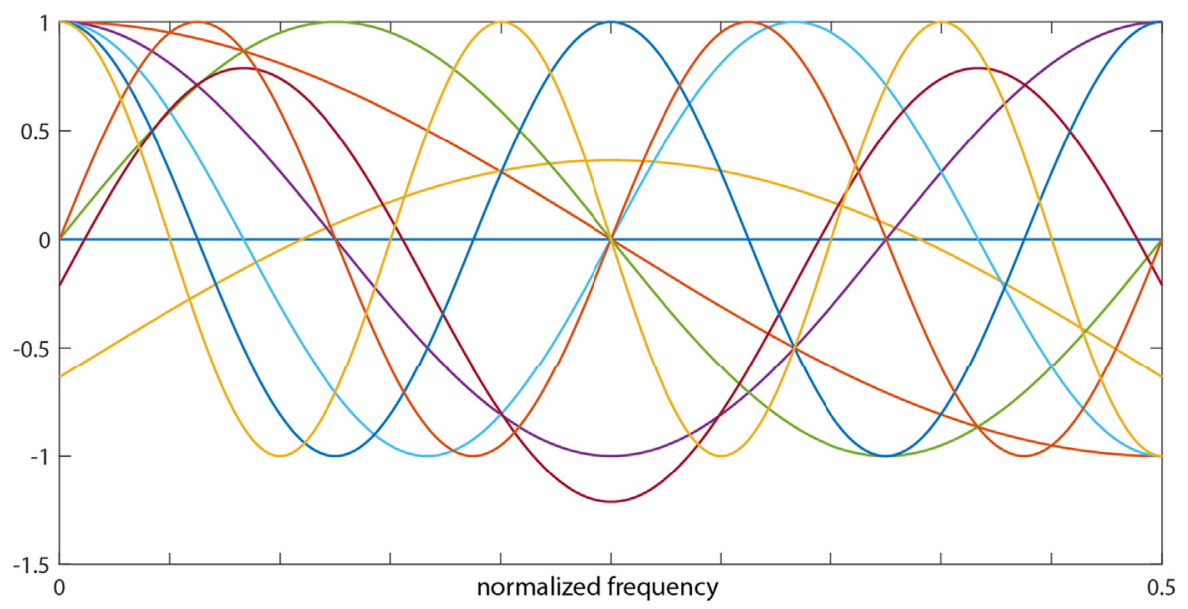

Fig. 2. Design of multi-filters with sinusoidal frequency gains as given in Eq. (25). 
upper limit of $\mathrm{J}$ is such that $J<B_{k j_{2}}^{\mathrm{BSS}} / \Delta f$ (there should not be oscillations shorter than the frequency resolution). The number $\mathrm{D}$ of filters used in GSM must be taken larger than or equal to 3 for the system of equation (17) to have an unique solution. Again, taking larger values of D will help in the presence of noise. The same upper limit applies as for the FD-BSS filters. Although the values of $\mathrm{J}$ and $\mathrm{D}$ may vary from one subband $B_{i}$ to another one, as simple choice is to set them invariant. Finally, the number $A_{i}$ of DA filters is the only one to depend explicitly on the subband $B_{i}$ since the aim is to turn an undetermined system $\left(N_{i}>M\right)$ into a (over)determined one, that is $\left(A_{i}+1\right) M \geq N_{i}$. However, because Eq. (21) is an approximation to Eq. (20) - with an approximation error that increases with the number of oscillations in $G_{j}^{\mathrm{DA}}(f)$ - it has been found that using more filters than required is detrimental. Therefore, $A_{i}=N_{i} / M-1$ is the recommended choice, where the ceiling operator $\lceil x\rfloor$ rounds to the least integer that is greater than or equal to $x$. In the following, the quantity $\left(A_{i}+1\right)$ will be referred to as the DA factor.

In conclusion, all multi-filters used in the proposed methodology can be designed automatically based on the aforementioned rules without requiring the user's intervention.

\subsection{Selection of frequency bands in FD-BSS}

The selection of frequency bands used in FD-BSS eventually ends up being the only demand on the user's intervention. It involves critical choices, yet it is also very flexible.

As explained in section 2, bands are defined by their lower and upper limits, $f_{L}^{i}$ and $f_{U}^{i}$, respectively, such that $B_{i}=\left[f_{L}^{i}, f_{U}^{i}\right]$. They should be selected narrow enough so that they include a small number of active modes and ideally satisfy the condition $N_{i} \leq M$. This is at the heart of the proposed methodology to separate modal contributions in the undetermined case. Consecutive bands $B_{i}, i=1, \ldots, I$ can be settled in an arbitrary way, with different bandwidths and they may possibly overlap. One precaution, however, is that the modes of interest are not cut by the band limits. In cases where this situation is difficult to meet (for instance in the presence of tight groups of modes), larger bands can be selected in conjunction with the use of DA which then requires the less stringent condition $N_{i} \leq M\left(A_{i}+1\right)$. One should also care that sufficient dynamics (i.e. in terms of signal-to-noise ratio) of the modes is included inside each band, as required for subsequent modal identification. Overall, a safe recommendation is to make sure that the bands contain the $3 \mathrm{~dB}$-bandwidth interval of each mode of interest.

Although some automated strategies may be conceived to automate the frequency axis division - such as systematic splitting into regularly spaced intervals - it is the authors' opinion that user's interaction is essential at this stage to manually select the appropriate bands by visual inspection. Not only is this action rather intuitive, but is also provides useful insights into the complete OMA process. The expertise requirement is no more demanding than the selection of modes from the stabilization diagram as commonly practiced in SSI-based OMA techniques.

\section{Experimental demonstrations}

The aim of this section is to validate the proposed methodology on synthetic and experimental data. First, two numerical examples are considered in order to assess estimation errors in various scenarios, including different types of damping and of signal-to-noise ratios (SNRs) and the presence of local mode shapes. Second, three experimental examples are considered in order to demonstrate the applicability of the methodology on real measurements; they are ordered with increasing degrees of difficulty corresponding to growing modal overlap, up to an extent that should theoretically invalidate the fundamental assumption of a diagonal spectral matrix in Eq. (5). The first two examples are laboratory experiments with low to medium modal overall, while the last example comprises in situ measurements with high modal overlap.

In order to allow reproducible research, care has been taken to use data that are freely available. The numerical examples have been introduced in Refs. [8] [29], while two sets of the experimental data come from a round-robin test organized on occasion of the IMAC XXVII 2011 conference [35].

\subsection{Numerical experiments}

\subsubsection{A simple three-degree-of-freedom system}

The aim of this first subsection is to test the proposed methodology, both in the determined and underdetermined configurations, on a simple three-degree-of-freedom mass-spring-damper system. This is an academic example already analyzed in Refs. [8,34]. The separation performance is first investigated with proportional damping, which has been theoretically shown to be favorable for BSS [12], and next with non-proportional damping, which is known to be more challenging. Finally, robustness to the presence of additive of noise is checked. In each case, the determined and underdetermined situations are considered.

\section{a) Proportional damping}

A configuration with proportional damping is first considered, with mass and stiffness matrices equal to 
Table 3

Parameter values used in the experiment.

\begin{tabular}{llc}
\hline & Determined case & Undetermined case \\
\hline Sampling frequency & & $F_{S}=10 \mathrm{~Hz}$ \\
Total number of modes & $M=3=3$ & $M=2$ \\
Number of channels & & {$[0 ; 1]$} \\
Bandwidths of FD-BSS filters & & $J=20$ \\
Number of FD-BSS filters & {$[0 ; 1]$} & $D=10$ \\
Bandwidths of GSM filters & $A=0$ & $A=1$ \\
Number of GSM filters & $\times$ & $p=2$ \\
Number of DA filters & & \\
Time shift in DA filters & & \\
\hline
\end{tabular}

Table 4

Identified modal parameters (proportional damping).

\begin{tabular}{|c|c|c|c|c|c|c|c|c|}
\hline \multirow[t]{2}{*}{$\alpha$} & \multirow[t]{2}{*}{ mode } & \multicolumn{3}{|c|}{ Frequency $(\mathrm{Hz})$} & \multicolumn{3}{|c|}{ Damping ratio (\%) } & \multirow[t]{2}{*}{ MOF (\%) } \\
\hline & & theoretical & determined & under determined & theoretical & determined & under determined & \\
\hline \multirow[t]{3}{*}{0.08} & 1 & 0.0895 & 0.0894 & 0.0896 & 7.11 & 7.08 & 7.01 & \\
\hline & 2 & 0.1458 & 0.1457 & 0.1482 & 4.37 & 4.37 & 4.67 & 9 \\
\hline & 3 & 0.2522 & 0.2522 & 0.2525 & 2.52 & 2.52 & 2.54 & 18 \\
\hline \multirow[t]{3}{*}{0.13} & 1 & 0.0895 & 0.0892 & 0.0907 & 11.55 & 11.48 & 11.36 & \\
\hline & 2 & 0.1458 & 0.1456 & 0.1425 & 7.10 & 7.10 & 7.13 & 15 \\
\hline & 3 & 0.2522 & 0.2522 & 0.2527 & 4.10 & 4.10 & 4.12 & 30 \\
\hline
\end{tabular}

$$
M=\left[\begin{array}{lll}
2 & 0 & 0 \\
0 & 1 & 0 \\
0 & 0 & 3
\end{array}\right], K=\left[\begin{array}{lll}
1 & 0 & 0 \\
0 & 1 & 0 \\
0 & 0 & 1
\end{array}\right]
$$

The damping matrix, $C=\alpha M$, is made proportional to the mass matrix with coefficient $\alpha=0.08,0.13$ that takes two values to account for different damping levels. This produces values of the modal overlaps factor in the range from 10 to $20 \%$ for $\alpha=0.08$ and $15-30 \%$ for $\alpha=0.13$ (see Table 4). Free displacement responses are synthesized with initial conditions $x(0)=\left[\begin{array}{lll}0 & 0 & 0\end{array}\right], \dot{x}(0)=\left[\begin{array}{lll}0 & 1 & 0\end{array}\right]$ and sampling frequency $F_{S}=10 \mathrm{~Hz}$. The determined case is synthesized by making all three outputs available, thus $M=N=3$. In this simple case, there is no need for dividing the frequency axis into subbands and therefore the full frequency range is taken for the FD-BSS filters up to $1 \mathrm{~Hz}$. Thus, $I=1$ and $B_{1, j}^{G C P}=1 \mathrm{~Hz}$. The underdetermined case is synthesized by removing the third output, giving $M=2$. DA is then used with $A_{1}=1$ filter by shifting data by $p=2$

(a)

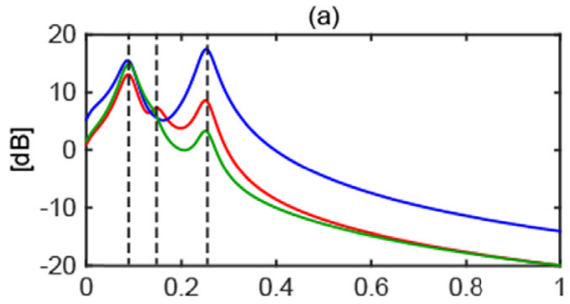

(c)

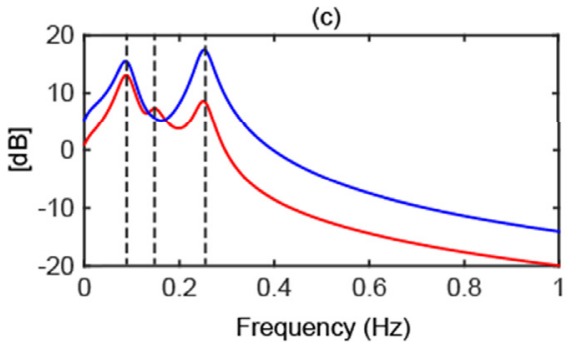

(b)

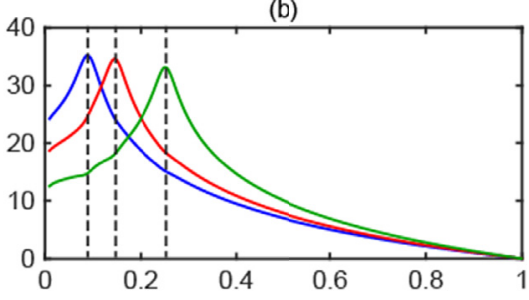

(d)

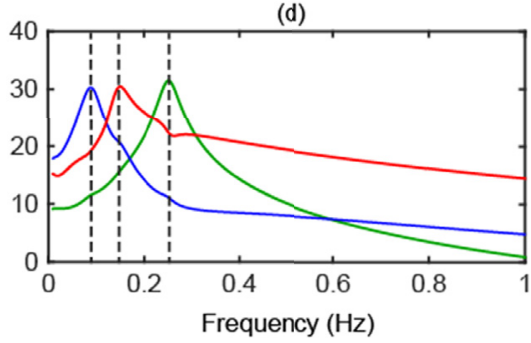

Fig. 3. Energy spectral densities of output measurements (first column) and of separated modal responses (second column) in the proportional damping case $(\Delta f=0.1 \mathrm{~Hz}, \alpha=0.08)$. a-b) Determined scenario $(M=3)$, c-d) underdetermined scenario $(M=2)$. Vertical black dotted lines indicate the theoretical locations of the resonance frequencies. 
samples, thus yielding $\left(A_{1}+1\right) \times M=4$ outputs in total (i.e. a DA factor of two), which is enough to identify a three-degreeof-freedom system. Finally, the modal parameters are identified with the GSM-based method of section 3 by designing filters in the full frequency range up to $1 \mathrm{~Hz}$. All filters have been designed with the sinusoidal gains advocated in subsection 5.2 (Eq. (25)). Table 3 resumes all the parameter values used in the experiment (as well as the numbers of FD-BSS and GSM filters).

Table 4 reports the values of the modal parameters identified after running the complete methodology (Table 2 of section 5 ) and compares them with the theoretical values. The energy spectral densities of the separated modal contributions $(\alpha=0.08)$ are shown in Fig. 3. It is seen that excellent separation and identification is achieved in the determined case. The identified modal parameters are very close to the theoretical values, with a maximum relative error of $0.1 \%$ on the natural frequency and $0.4 \%$ on damping ratio for $\alpha=0.08$ and $0.3 \%$ on the natural frequency and $0.6 \%$ on damping ratio for $\alpha=0.13$ in the determined case. As expected, the undetermined case is more difficult to solve since it faces a deficit of information, yet the errors remain small: the maximum relative error is $1.6 \%$ on the natural frequencies and $6.9 \%$ on damping ratios for $\alpha=$ 0.08 and $2.3 \%$ on the natural frequencies and $1.7 \%$ on damping ratios for $\alpha=0.13$. Although Fig. 3 (d) shows that the mass lines (asymptotic behavior when $f \gg f_{k}$ ) of the modal responses are somewhat distorted, this error seems to moderately affect the modal identification process, provided that the latter is performed in the restricted 3-dB bandwidth as recommended in subsection 5.2 .

\section{b) Non-proportional damping}

The configuration with non-proportional damping is now investigated. This configuration is known to be more challenging because it involves complex modes which are not resolved by BSS methods such as SOBI [11,12] or CP [16]. However, this should not be a limitation of FD-BSS since the optimization problem (10) is solved for complex-valued matrices.

The system matrices are set as follows,

$$
M=\left[\begin{array}{lll}
1 & 0 & 0 \\
0 & 2 & 0 \\
0 & 0 & 1
\end{array}\right], K=\left[\begin{array}{ccc}
5 & -1 & 0 \\
-1 & 4 & -3 \\
0 & -3 & 3.5
\end{array}\right], \quad C=\left[\begin{array}{ccc}
0.0894 & -0.0084 & 0.0003 \\
-0.0084 & 0.1301 & -0.0244 \\
0.0003 & -0.0244 & 0.0772
\end{array}\right]
$$

and the same initial conditions as before are used to synthesize free displacement vibrations. Table 5 reports the values of the modal parameters identified after separation and Fig. 4 displays the spectral densities of the separated modal contributions for the determined and underdetermined cases. Overall, excellent results are obtained, even for the last two modes that highly overlap (MOF of 50\%). The maximum relative error is $0.1 \%$ on the natural frequencies and $0 \%$ on the damping ratios for the determined case and.0.6\% on the natural frequencies and $4 \%$ on the damping ratios for the determined case. The separation of modal responses in the underdetermined configuration with DA seems even better than with proportional damping, even though the slope of the mass lines (asymptotic behavior when $f \gg f_{k}$ ) is again underestimated.

Finally, white Gaussian noise is added to the measurements in order to test the robustness of the algorithm. The root mean square (RMS) value of the noise is set to $5 \%, 10 \%, 15 \%, 20 \%$ of the signal RMS value, which corresponds to SNRs of $26 \mathrm{~dB}, 20 \mathrm{~dB}$, $16 \mathrm{~dB}$, and $14 \mathrm{~dB}$, respectively. Tables 6 and 7 list the identified estimated modal parameters after separation as a function of the different SNRs for the proportional and non-proportional configurations, respectively. It is checked that excellent robustness to noise is achieved.

\subsubsection{A 15-degree-of-freedom system}

The methodology is now tested on a simulated, but realistic, 15-deegree-of-freedom system. This example has been regularly used in other publications to test the capability of BSS algorithms and has been intentionally designed to produce some modes which are complex, heavily damped, strongly coupled modes, as well as local (see e.g. Refs. [12,29]). It is investigated here to check the algorithm capability to deal with a more severely undetermined configuration than in the previous three-degree-of-freedom example.

The system comprises discrete parameters, as illustrated in Fig. 5. Masses $1-10$ have value $m_{1}=10 / 386.09 \mathrm{~kg}$ and masses $11-15$ value $\mathrm{m} 2=0.5 / 386.09 \mathrm{~kg}$; all springs have value $k=1000 \mathrm{~N} / \mathrm{m}$. The dampers in the first column connecting masses $6-10$ have damping value $c_{1}=0.20 \mathrm{~kg} / \mathrm{s}$, those in the second column connecting masses $6-10$ to masses $1-5$ have damping values $c_{2}=0.20 \mathrm{~kg} / \mathrm{s}$, those in the third column connecting masses $1-5$ have damping value $c_{1}$ and those in the fourth column connecting masses $11-15$ to masses $1-5$ have damping value $c_{3}=0.05 \mathrm{~kg} / \mathrm{s}$.

Table 5

Identified modal parameters (non-proportional damping).

\begin{tabular}{|c|c|c|c|c|c|c|c|}
\hline \multirow[t]{2}{*}{ mode } & \multicolumn{3}{|c|}{ Frequency $(\mathrm{Hz})$} & \multicolumn{3}{|c|}{ Damping ratio (\%) } & \multirow[t]{2}{*}{$\operatorname{MOF}(\%)$} \\
\hline & theoretical & determined & under determined & theoretical & determined & under determined & \\
\hline 1 & 0.1039 & 0.1039 & 0.1045 & 4.00 & 4.00 & 4.01 & \\
\hline 2 & 0.3425 & 0.3423 & 0.3445 & 2.00 & 2.00 & 2.08 & 4 \\
\hline 3 & 0.3713 & 0.3712 & 0.3729 & 2.00 & 2.00 & 1.98 & 50 \\
\hline
\end{tabular}


(a)

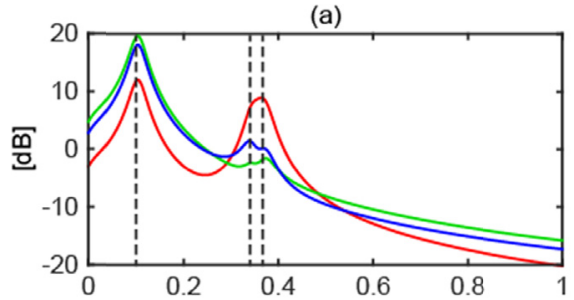

(c)

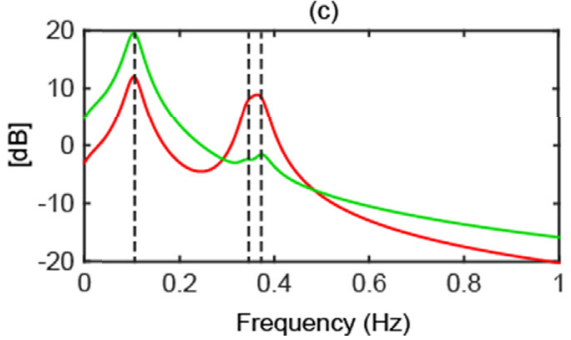

(b)

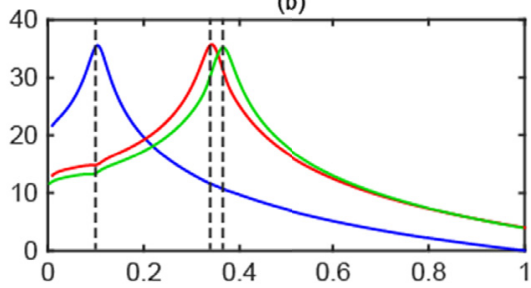

(d)

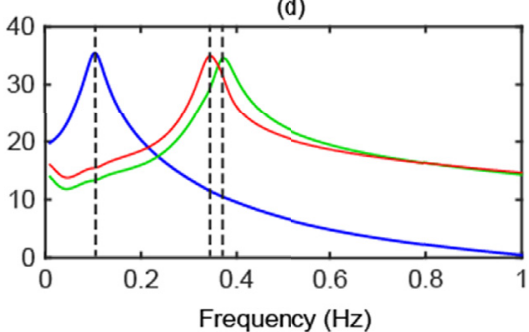

Fig. 4. Energy spectral densities of output measurements (first column) and of separated modal responses (second column) in the non-proportional damping case $(\Delta f=0,1 \mathrm{~Hz})$. a-b) Determined scenario $(M=3), \mathrm{c}-\mathrm{d})$ underdetermined scenario $(M=2)$. Vertical black dotted lines indicate the theoretical locations of the resonance frequencies.

c) Robustness to noise

Table 6

Identified modal parameters for various noise levels (proportional damping).

\begin{tabular}{|c|c|c|c|c|c|c|c|c|c|c|}
\hline \multirow[t]{2}{*}{ mode } & \multicolumn{5}{|c|}{ Frequency (Hz) } & \multicolumn{5}{|c|}{ Damping ratio (\%) } \\
\hline & $0 \%$ & $5 \%$ & $10 \%$ & $15 \%$ & $20 \%$ & $0 \%$ & $5 \%$ & $10 \%$ & $15 \%$ & $20 \%$ \\
\hline 1 & 0.0894 & 0.0894 & 0.0894 & 0.0894 & 0.0894 & 7.08 & 7.09 & 7.14 & 7.07 & 7.01 \\
\hline 2 & 0.1457 & 0.1457 & 0.1457 & 0.1456 & 0.1457 & 4.37 & 4.36 & 4.11 & 4.26 & 4.33 \\
\hline 3 & 0.2522 & 0.2522 & 0.2522 & 0.2522 & 0.2522 & 2.52 & 2.54 & 2.52 & 2.54 & 2.54 \\
\hline
\end{tabular}

Table 7

Identified modal parameters for various noise levels (non-proportional damping).

\begin{tabular}{|c|c|c|c|c|c|c|c|c|c|c|}
\hline \multirow[t]{2}{*}{ mode } & \multicolumn{5}{|c|}{ Frequency (Hz) } & \multicolumn{5}{|c|}{ Damping ratio (\%) } \\
\hline & $0 \%$ & $5 \%$ & $10 \%$ & $15 \%$ & $20 \%$ & $0 \%$ & $5 \%$ & $10 \%$ & $15 \%$ & $20 \%$ \\
\hline 1 & 0.1039 & 0.1039 & 0.1039 & 0.1040 & 0.1040 & 4.00 & 4.00 & 4.00 & 3.99 & 4.00 \\
\hline 2 & 0.3423 & 0.3424 & 0.3422 & 0.3422 & 0.3423 & 2.00 & 1.94 & 1.89 & 1.90 & 1.88 \\
\hline 3 & 0.3712 & 0.3712 & 0.3712 & 0.3712 & 0.3710 & 2.00 & 1.99 & 1.93 & 1.69 & 1.85 \\
\hline
\end{tabular}

The system is excited by random forces having unit magnitude spectra and uniformly random phases and its responses are obtained at all degrees of freedom $(M=15)$. All signals are sampled at $F_{\mathrm{S}}=1024 \mathrm{~Hz}$ and the correlation matrix CSM is computed on a 163840 sample-long record.

Three configurations are investigated. The first one is with $M=15$ measurements, which leads to a determined full band analysis. The second one is with a limited number of $M=10$ measurements. In order to deal with this underdetermined case, the frequency axis is divided into two bands $[0 ; 100] \mathrm{Hz}$ and $[100 ; 400] \mathrm{Hz}$, where care is taken to include no more than 10 modes in each band. This configuration is then solved without DA. The last configuration is with only $M=5$ channels. Two strategies are possible at this stage: the first one is to further divide the frequency axis into narrower subbands and the second one is to use DA. However, because the band $[40 ; 100] \mathrm{Hz}$ seems to contain a group of modes with very high modal overlap, the second strategy is preferred. It leads to two frequency bands $[0 ; 100] \mathrm{Hz}$ and $[100 ; 400] \mathrm{Hz}$ which contain no more than 10 and 5 modes, respectively. Therefore, one DA filter is used in the first band in order the double the dimension of the observations. The parameter values used in the experiment are resumed in Table 8. The power spectral densities (PSD) of the 


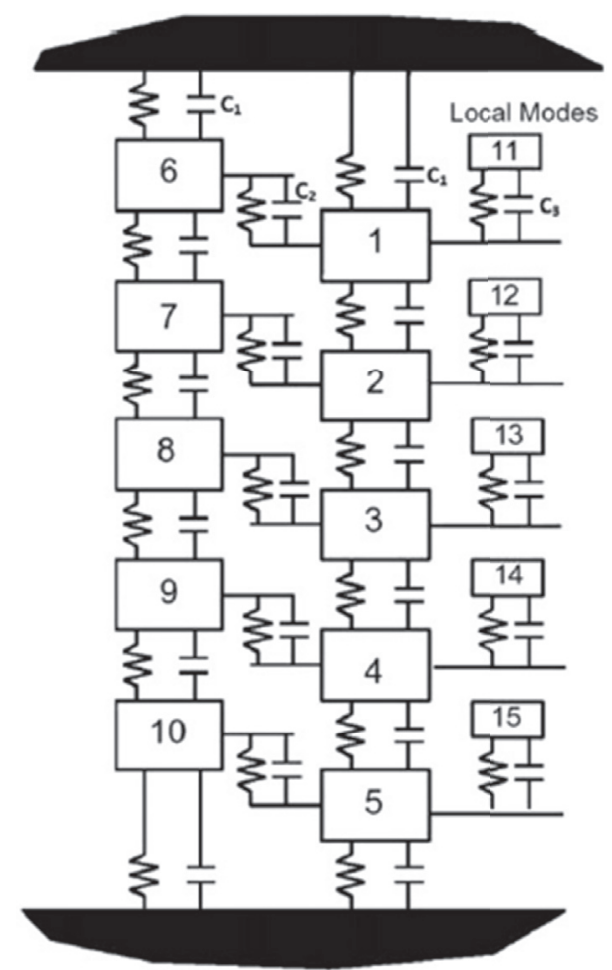

Fig. 5. Simulated 15-degree-of-freedom system.

Table 8

Parameter values used in the experiment.

\begin{tabular}{|c|c|c|c|}
\hline & Determined case & Undetermined case 1 & Undetermined case 2 \\
\hline Sampling frequency & & $F_{s}=1024 \mathrm{~Hz}$ & \\
\hline Total number of modes & & $N=15$ & \\
\hline Number of sensors & $M=15$ & $M=10$ & $M=5$ \\
\hline Bandwidths of FD-BSS filters & {$[0 ; 400]$} & {$[0 ; 100],[100 ; 400]$} & {$[0 ; 100],[100 ; 400]$} \\
\hline Number of modes in bands & 15 & 10,5 & 10,5 \\
\hline Number of FD-BSS filters & & $J=20$ & \\
\hline Bandwidths of GSM filters & & 3-dB bandwidth & \\
\hline Number of GSM filters & & $D=10$ & \\
\hline Number of DA filters & $A=0$ & $A=0$ & $A_{1}=1, A_{2}=0$ \\
\hline Time shift in DA filters & $x$ & $\times$ & $p=2$ \\
\hline Dimension of augmented data & & & 10,5 \\
\hline
\end{tabular}

separated modal contributions are displayed in Fig. 6 for all tested configurations. Table 9 collects the values of the identified modal parameters.

Overall, excellent results are obtained, which are consistent with those obtained in previous references (e.g. Refs. $[12,29,34])$. Although DA introduces slight distortions in the mass and stiffness lines of the separated mode spectra (see Fig. 6(d)) as already observed in the previous example, the identification of the modal parameters given in the last line of Table 9 remains very good as seen. The relative error on the natural frequencies is less than $1 \%$ on the average; the relative error on the damping ratios is about 3\% without DA and 7\% with DA. It is noteworthy that the two very close modes at 53.32 and $53.30 \mathrm{~Hz}$ have been correctly separated in all cases.

It is noteworthy that this example also demonstrates that the proposed methodology easily makes the difference between structural and numerical modes. This is well illustrated in the second frequency band of Fig. 6(c) where ten channels are used to separate five modes. Although the system is now overdetermined, the five structural modes are correctly extracted and the five residual ones appear as flat spectra without any resonance (in grey color in Fig. 6(c)). This is a most desirable property, which indeed appears to be not shared by all BSS methods. 
(a)

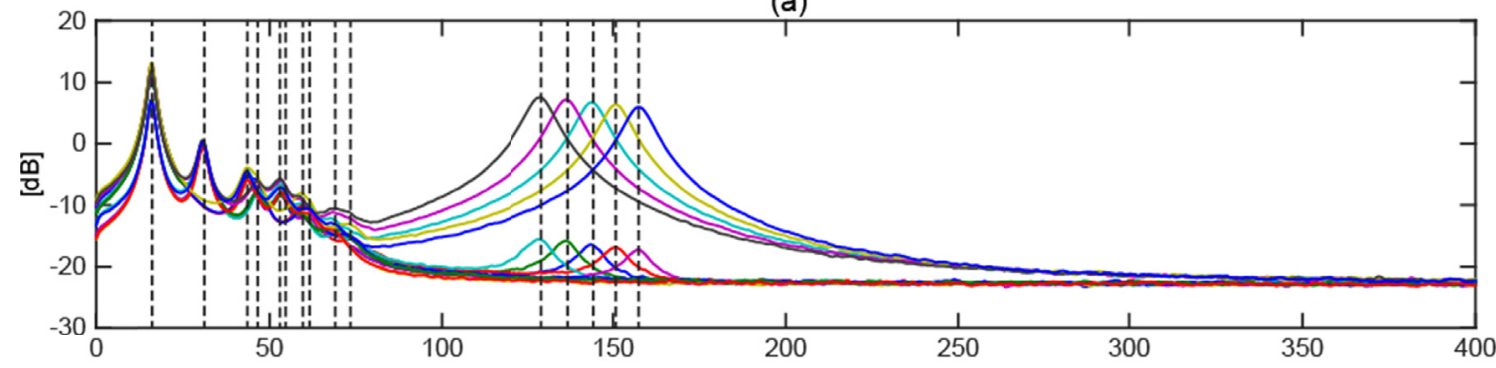

(b)

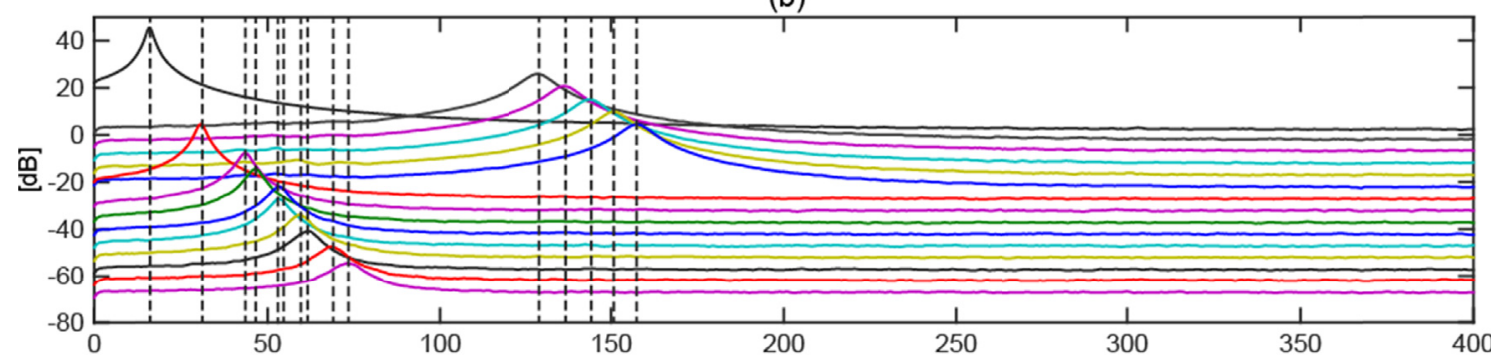

(c)

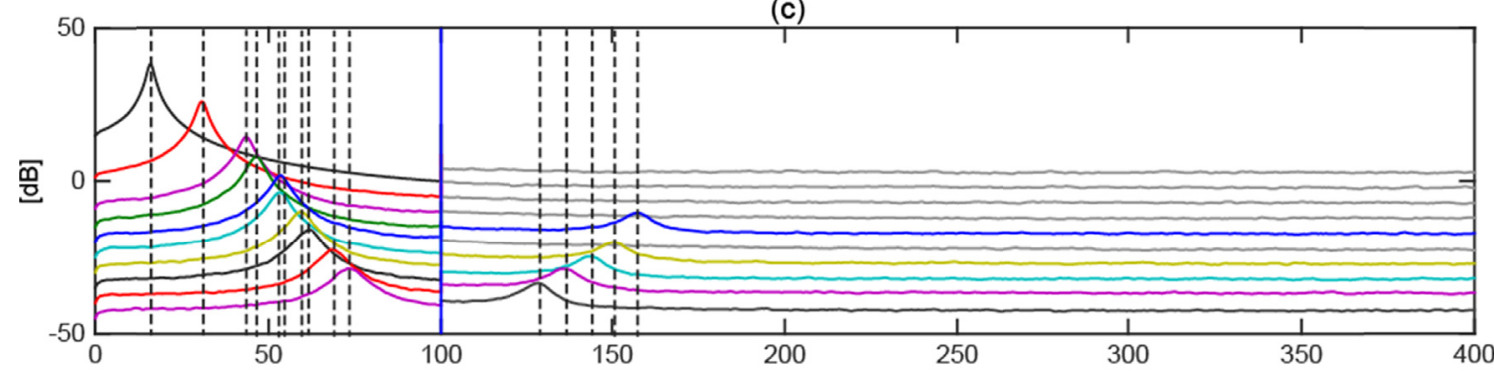

(d)

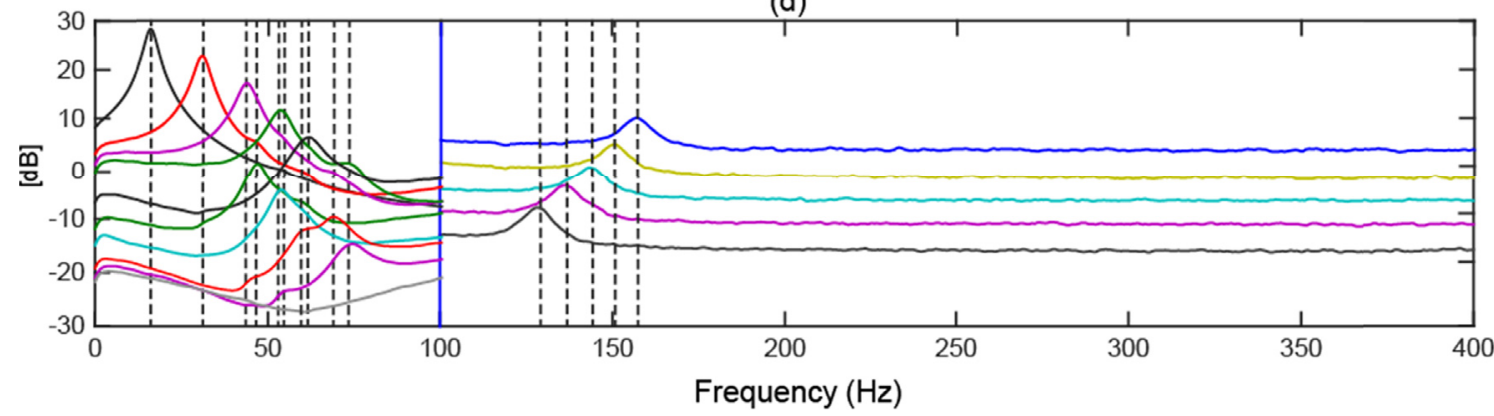

Fig. 6. Power spectral densities of a) output measurements and of separated modal responses using b) 15 channels, c) 10 channels and 2 subbands and d) 5 channels and 2 subbands with DA in the first one $(\Delta f=0.5 \mathrm{~Hz})$. Vertical black dotted lines indicate the theoretical locations of the resonance frequencies.

\subsection{Real data}

The following sections now demonstrate the application of the proposed methodology on three real data sets. The first two data sets come from laboratory experiments whereas the third one originates from an in situ experiment. They are organized hereafter with increasing modal overlap and, therefore, with increasing degrees of complexity for BSS.

\subsubsection{Circular plate}

The vibration response of a lightly damped aluminum circular plate is first considered. The device under test is shown in Fig. 7 and was investigated in Ref. [29]. The structure is excited with white Gaussian noise and the response is recorded by 30 uniaxial accelerometers at a sampling frequency of $1600 \mathrm{~Hz}$ for $5 \mathrm{~min}$. 
Table 9

Identified modal parameters.

\begin{tabular}{|c|c|c|c|c|c|c|c|}
\hline \multicolumn{4}{|l|}{ Natural frequencies (Hz) } & \multicolumn{4}{|c|}{ Damping ratio (\%) } \\
\hline \multirow[b]{2}{*}{ True } & \multicolumn{3}{|c|}{ Number of channels } & \multirow[b]{2}{*}{ True } & \multicolumn{3}{|c|}{ Number of channels } \\
\hline & $\overline{15}$ & 10 & 5 & & $\overline{15}$ & 10 & 5 \\
\hline 15.99 & 15.99 & 16.00 & 15.93 & 1.00 & 1.22 & 1.18 & 1.19 \\
\hline 30.86 & 30.84 & 30.85 & 30.67 & 1.94 & 1.89 & 1.88 & 1.95 \\
\hline 43.60 & 43.62 & 43.62 & 43.28 & 2.74 & 2.63 & 2.67 & 2.85 \\
\hline 46.44 & 46.49 & 46.48 & 46.71 & 2.91 & 2.94 & 2.94 & 3.03 \\
\hline 53.32 & 53.36 & 53.36 & 52.93 & 3.34 & 3.21 & 3.42 & 3.96 \\
\hline 53.39 & 53.42 & 53.54 & 54.02 & 3.35 & 3.20 & 3.27 & 3.37 \\
\hline 59.41 & 59.45 & 59.52 & 59.17 & 3.72 & 3.62 & 3.74 & 4.60 \\
\hline 61.62 & 61.67 & 61.70 & 62.53 & 3.86 & 3.89 & 3.86 & 4.28 \\
\hline 68.81 & 68.83 & 69.01 & 69.77 & 4.30 & 3.90 & 4.01 & 4.39 \\
\hline 73.63 & 73.53 & 73.83 & 74.81 & 4.59 & 4.33 & 4.63 & 5.27 \\
\hline 128.84 & 128.87 & 128.13 & 128.10 & 2.61 & 2.62 & 2.65 & 2.64 \\
\hline 136.55 & 136.60 & 135.95 & 135.93 & 2.46 & 2.46 & 2.41 & 2.40 \\
\hline 143.86 & 143.90 & 143.28 & 143.26 & 2.33 & 2.33 & 2.36 & 2.36 \\
\hline 150.83 & 150.88 & 150.27 & 150.26 & 2.22 & 2.21 & 2.26 & 2.26 \\
\hline 157.47 & 157.51 & 156.98 & 156.97 & 2.12 & 2.13 & 2.10 & 2.10 \\
\hline Mean relative error (\%) & 0.06 & 0.23 & 0.75 & & 3.85 & 3.01 & 7.06 \\
\hline
\end{tabular}

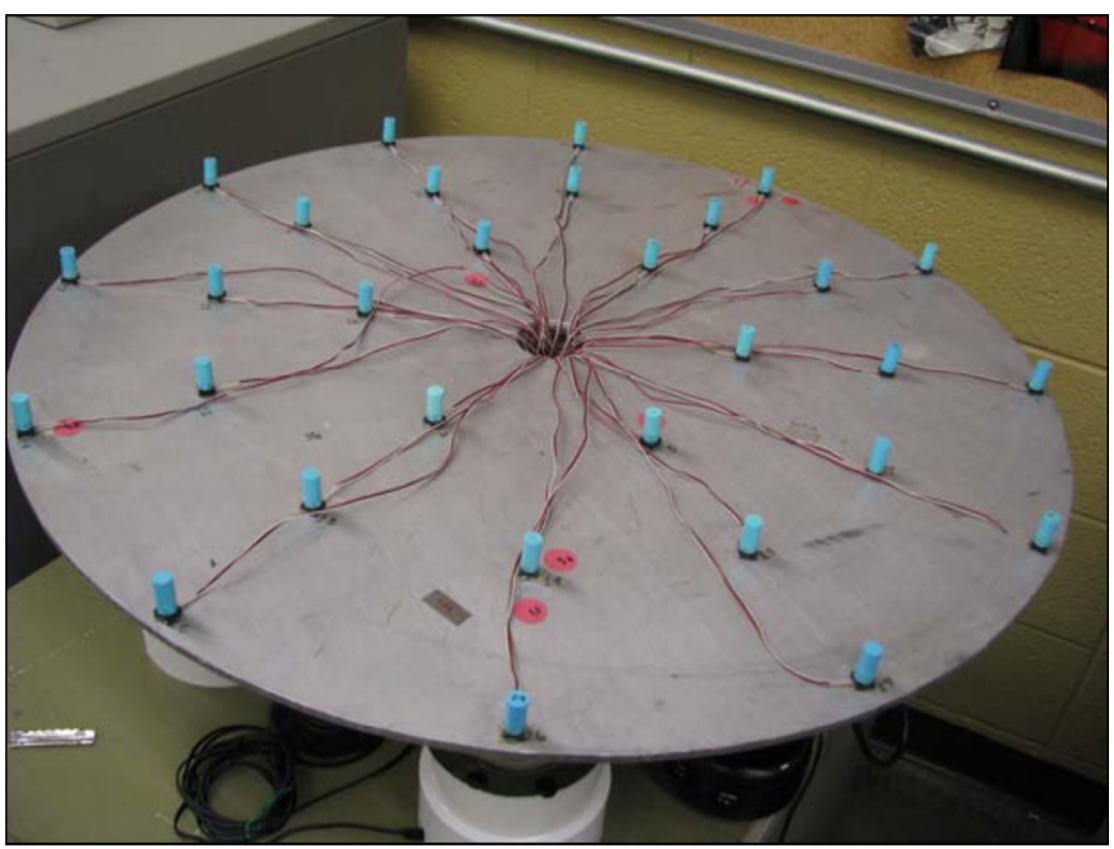

Fig. 7. Experimental setup for a lightly damped circular plate.

The frequency range of interest is [10; 800] Hz. Inspection of the response PSDs in Fig. 8(a) indicates that there are at least 17 modes in this frequency range. Therefore, FD-BSS is first used in full band with the total number of available channels, $M=30$ and $J=20$ frequency gains. Fig. 8(b) displays the separation results where it is noted that several modes are not well separated, probably because their number appears aposteriori greater than first expected (23 modes are now seen) and thus the problem is actually underdetermined. By splitting the frequency axis into two bands, $[0 ; 400] \mathrm{Hz}$ and $[400 ; 800] \mathrm{Hz}$, the separation results are greatly improved as shown in Fig. 8(c). In particular, it is noteworthy that there exists singular modes which coexist at the same frequencies due to the circular symmetry of the structure - which are perfectly well separated (e.g. modes around $57 \mathrm{~Hz}, 133 \mathrm{~Hz}, 222 \mathrm{~Hz}, 232 \mathrm{~Hz}$, etc). The values of the identified modal parameters are listed in Table 10.

In order to demonstrate the separation capability of the proposed methodology, the same analysis is repeated while progressively reducing the number of channels and, consequently, splitting the frequency axis in smaller bands and applying DA when necessary. The configurations are resumed in the legend of Fig. 9 which displays the separation results. The values of the identified modal parameters are reported in Table 11. Overall, it is seen that results are very close to the previous ones even 

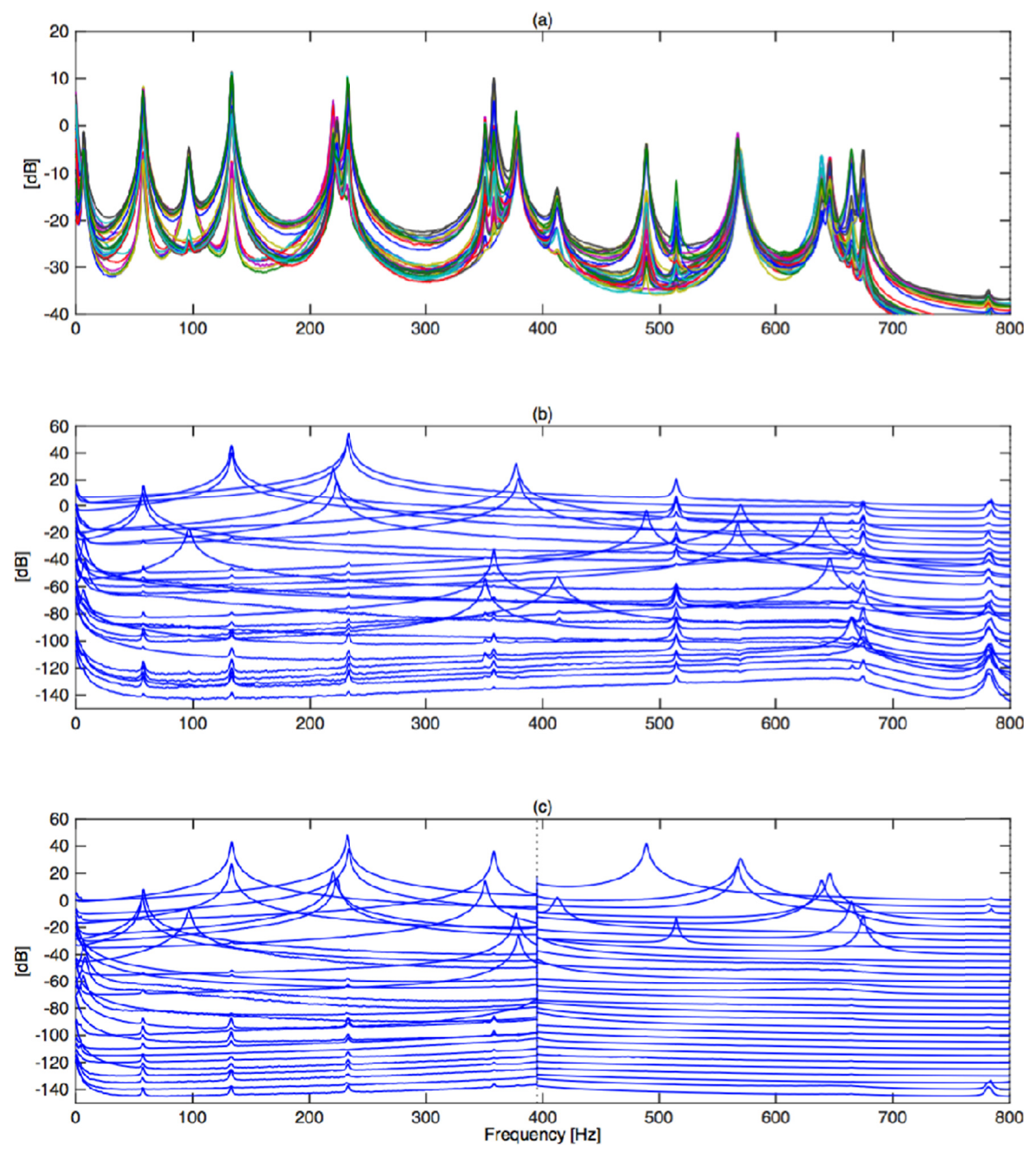

Fig. 8. Power spectral densities of a) output measurements and of separated modal responses with b) $M=30$ channels and c) $M=15$ channels and 2 subbands.

\section{Table 10}

Identified modal parameters (natural frequencies and damping ratios).

\begin{tabular}{llllll}
\hline Freq. (Hz) & Damp. (\%) & Freq. (Hz) & Damp. (\%) & Freq. (Hz) & Damp. (\%) \\
\hline 57.01 & 0.07 & 233.32 & 0.06 & 513.91 & 567.56 \\
57.82 & 0.17 & 350.90 & 0.12 & 569.91 & 0.05 \\
96.47 & 0.57 & 358.29 & 0.04 & 639.22 & 0.08 \\
132.85 & 0.05 & 377.28 & 0.08 & 646.01 & 0.15 \\
132.88 & 0.03 & 379.19 & 0.10 & 664.72 & 0.14 \\
219.67 & 0.11 & 412.45 & 0.30 & 674.48 & 0.09 \\
222.90 & 0.11 & 414.24 & 0.22 & \\
231.96 & 0.06 & 488.46 & 0.08 & & 0.05 \\
\hline
\end{tabular}


(a)

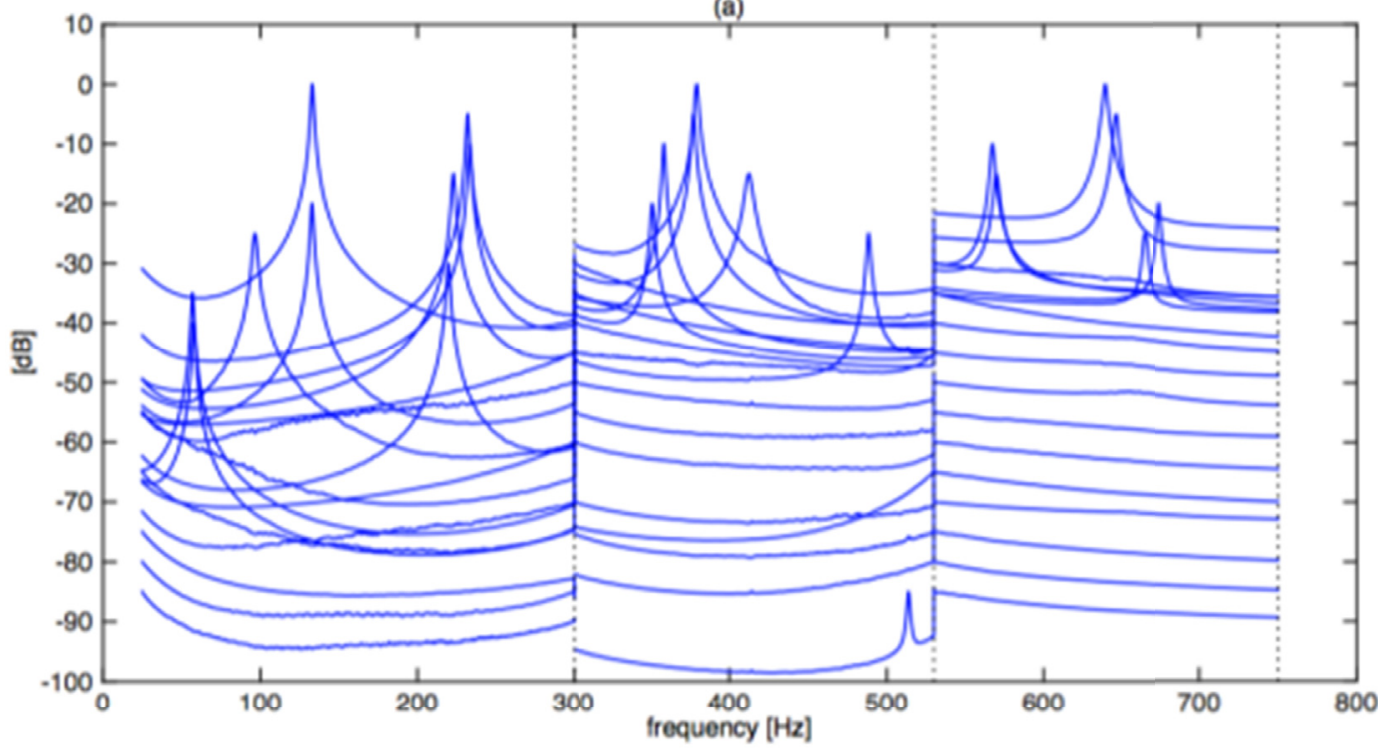

(b)

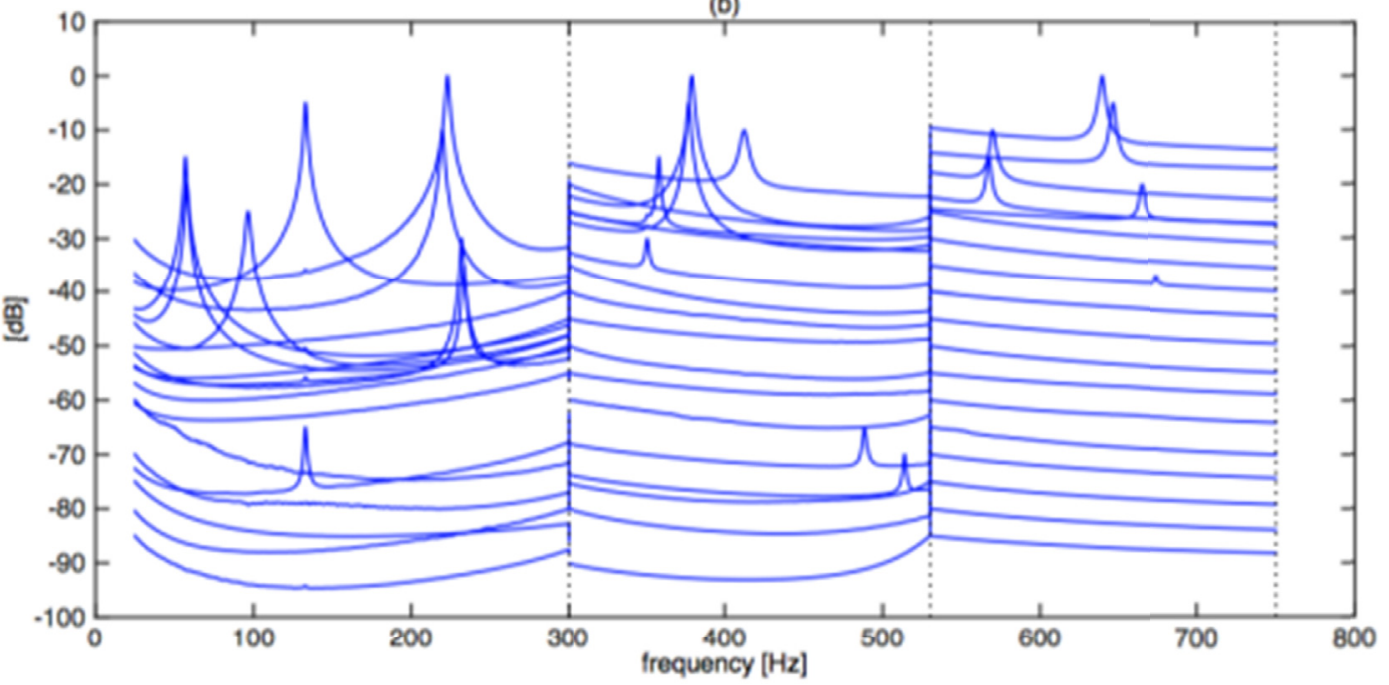

(c)

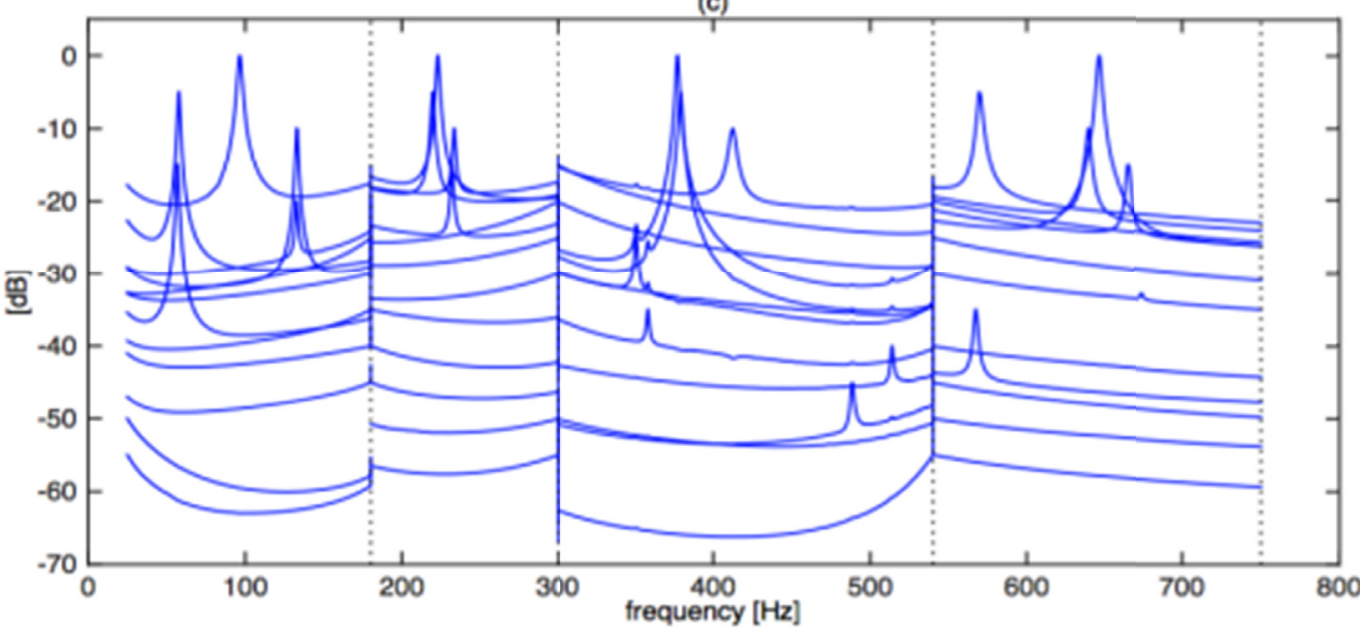

Fig. 9. Power spectral densities of separated modal responses obtained under three different configurations: a) $M=9$ channels, 3 bands [10; 300] Hz, [300; 525] $\mathrm{Hz}$ and [525; 750] Hz and DA factor $=2$, b) $M=6$ channels, 3 bands [10; 300] Hz, [300; 525] Hz and [525; 750] Hz and DA factor $=3$ and c) $M=4$ channels, 4 bands $[10 ; 175] \mathrm{Hz},[175 ; 300] \mathrm{Hz},[300 ; 525] \mathrm{Hz}$ and $[525 ; 750] \mathrm{Hz}$ and DA factor $=3$. 
Table 11

Identified modal parameters (natural frequencies and damping ratios) using reduced numbers of channels and data augmentation.

\begin{tabular}{|c|c|c|c|c|c|}
\hline \multicolumn{2}{|c|}{$2 \times 9$ channels } & \multicolumn{2}{|c|}{$3 \times 6$ channels } & \multicolumn{2}{|c|}{$3 \times 4$ channels } \\
\hline Freq. (Hz) & Damp. (\%) & Freq. $(\mathrm{Hz})$ & Damp. (\%) & Freq. $(\mathrm{Hz})$ & Damp. (\%) \\
\hline 56.95 & 0.05 & 56.94 & 0.03 & 56.97 & 0.08 \\
\hline 57.80 & 0.11 & 57.80 & 0.11 & 57.81 & 0.13 \\
\hline 96.46 & 0.63 & 96.47 & 0.64 & 96.38 & 0.38 \\
\hline 132.79 & 0.07 & 132.87 & 0.04 & 132.83 & 0.03 \\
\hline 132.93 & 0.06 & 132.90 & 0.06 & 132.90 & 0.04 \\
\hline 219.69 & 0.09 & 219.68 & 0.11 & 219.65 & 0.09 \\
\hline 222.90 & 0.13 & 222.90 & 0.13 & 222.90 & 0.13 \\
\hline 231.97 & 0.04 & 231.97 & 0.04 & - & - \\
\hline 233.32 & 0.05 & 233.34 & 0.06 & 233.23 & 0.04 \\
\hline 350.89 & 0.07 & 350.91 & 0.08 & 350.84 & 0.09 \\
\hline 358.25 & 0.04 & - & - & 358.24 & 0.07 \\
\hline 377.24 & 0.07 & 377.25 & 0.07 & 377.25 & 0.07 \\
\hline 379.14 & 0.10 & 379.15 & 0.10 & 379.14 & 0.10 \\
\hline 412.42 & 0.19 & 412.55 & 0.18 & 412.23 & 0.18 \\
\hline 488.43 & 0.06 & 488.49 & 0.07 & 488.60 & 0.07 \\
\hline 513.80 & 0.05 & 513.79 & 0.05 & 513.87 & 0.08 \\
\hline 514.14 & 0.06 & - & - & - & - \\
\hline 567.50 & 0.07 & 567.49 & 0.07 & 567.51 & 0.07 \\
\hline 570.03 & 0.07 & 570.20 & 0.04 & 570.18 & 0.03 \\
\hline 639.26 & 0.11 & 639.23 & 0.10 & 639.24 & 0.11 \\
\hline 646.08 & 0.09 & 646.07 & 0.08 & 646.06 & 0.07 \\
\hline 664.79 & 0.04 & 664.76 & 0.04 & 664.73 & 0.06 \\
\hline 674.49 & 0.05 & 674.58 & 0.05 & 674.69 & 0.06 \\
\hline
\end{tabular}

by using as few as four channels to separate 23 modes (only two very closely spaced modes could not be identified in this last setting).

\subsubsection{Scaled model of a wind turbine blade}

The second experimental data come from a scaled model of a wind turbine blade [35]. The structure is fixed at its root (as shown in Fig. 10) and it is excited by means of random tapping for duration of about 5 min. The vibration response is measured in all three directions with triaxial accelerometers at 16 locations at a sampling rate of $F_{\mathrm{s}}=512 \mathrm{~Hz}$. The frequency range of study is up to $200 \mathrm{~Hz}$. In the following, only the dynamical behavior in the vertical direction is considered for the analysis, which amounts to $M=16$ channels. As seen in Fig. 11(a), the PSDs of the measured responses indicate the presence of significant modal overlap and of a high modal density, in particular in the higher frequency range. This suggests a number of modes much greater than the number of available channels.

At first, all $16 \mathrm{z}$-channels are considered and the frequency axis is divided into four bands, [2; 68] Hz, [68; 122] Hz, [122; $170] \mathrm{Hz}$, and [170; 256] Hz. However, due to the high modal density, some modes are inevitably close to the chosen band limits. In order to resolve them, another analysis is conducted at the same time with the configuration: [0; 54] Hz, [54; 100] $\mathrm{Hz},[100 ; 150] \mathrm{Hz}$, and $[150 ; 256] \mathrm{Hz}$. This will make possible to correctly resolve the modes around the band limits of the first configuration. Results of the separation are displayed in Fig. 11(b-c) and effectively show that the second configuration

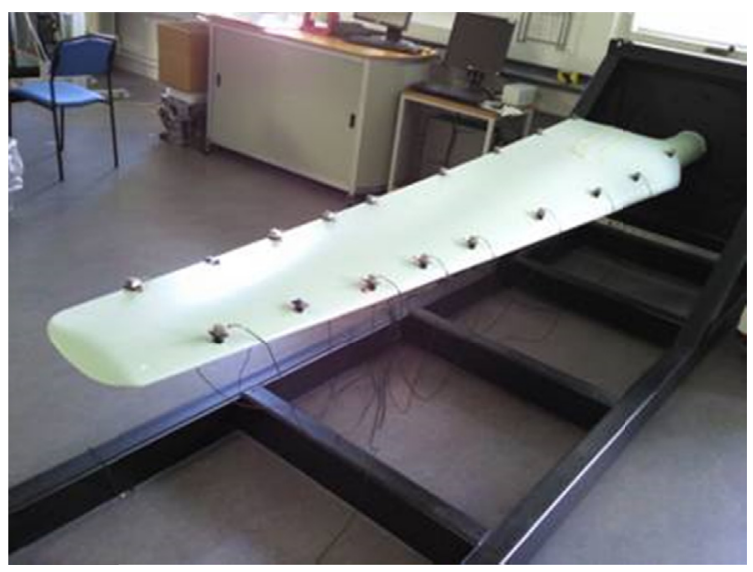

Fig. 10. Scaled model of a wind turbine blade. 
(a)

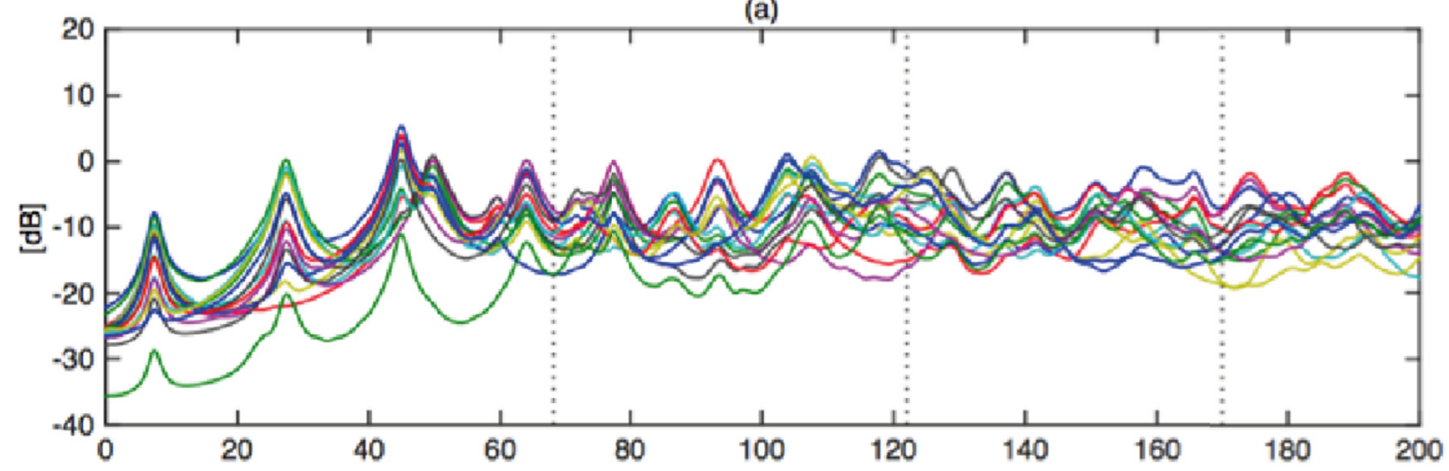

(b)

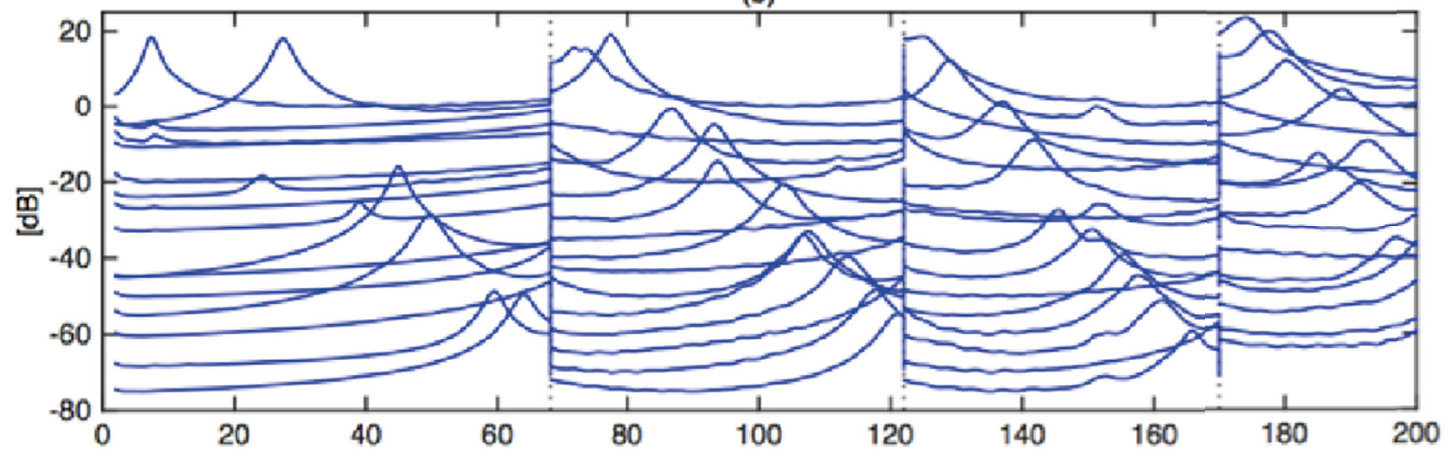

(c)

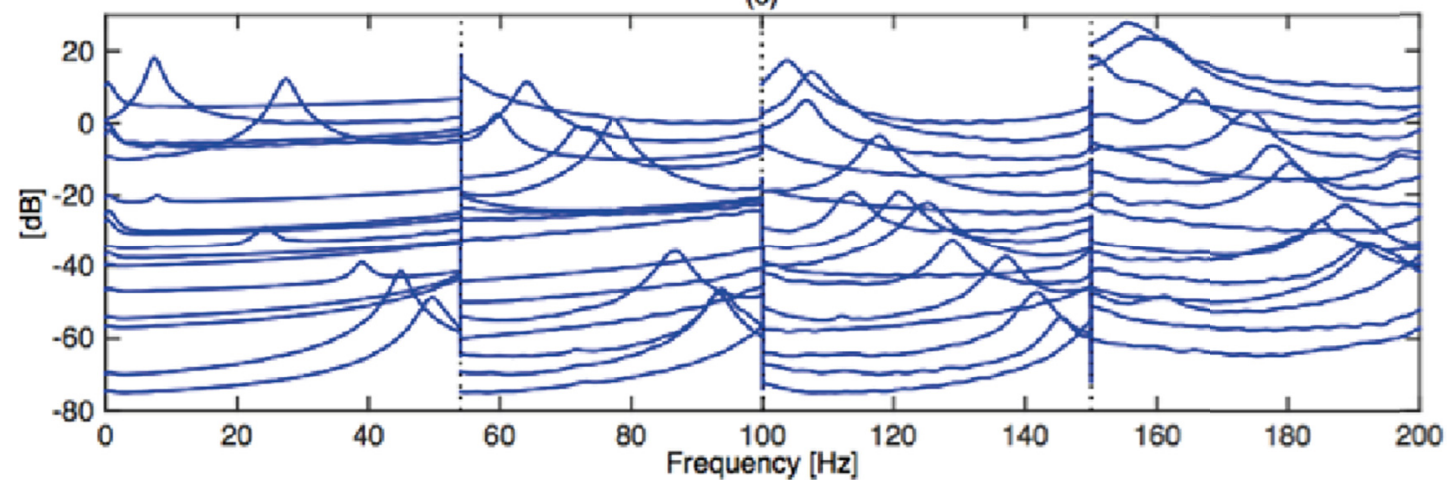

Fig. 11. Power spectral densities of a) output measurements of the wind turbine blade and of b-c) separated modal responses with two different but complimentary configurations based on four subbands ( $M=16$ channels).

complements the first one. Overall, all modes are correctly separated, even in the frequency region of highest modal density. Table 12 lists the values of the identified modal parameters together with the respective MOFs. It is remarkable that separation could be achieved for modes with MOFs greater than $100 \%$, despite the working assumption of BSS requiring negligible coupling between modes. This demonstrates the robustness of the proposed FD-BSS algorithm.

Next, the situation is considered in which not all available channels are used in the algorithm, but only $M=8$, with the same subband configurations as before. Obviously, the number of active modes in each band is now higher than the number of available channels, especially in the higher part of the spectrum where modal density is high, and thus DA is necessary. Therefore, eight extra channels are generated by shifting the original data by two samples with one DA filter. The separated spectra are shown in Fig. 12 and the corresponding identified modal parameters are listed in Table 13. It can be checked that, overall, the results are well consistent with those found previously by using twice as many channels. This is further checked by comparing the first sixth reconstructed modes shapes in the two configurations, as shown in Fig. 13. Although torsional 
Table 12

Identified modal parameters together with the estimated modal overlap factors ( $M=16$ channels).

\begin{tabular}{|c|c|c|c|c|c|c|c|c|}
\hline Freq. (Hz) & Damp. (\%) & MOF & Freq. (Hz) & Damp. (\%) & MOF & Freq. (Hz) & Damp. (\%) & MOF \\
\hline 7.33 & 1.19 & $1 \%$ & 106.52 & 1.13 & $119 \%$ & 155.50 & 0.85 & $77 \%$ \\
\hline 27.27 & 1.35 & $5 \%$ & 107.64 & 0.91 & $56 \%$ & 158.89 & 1.01 & $121 \%$ \\
\hline 38.94 & 1.71 & $15 \%$ & 113.55 & 0.94 & $42 \%$ & 160.81 & 0.85 & $80 \%$ \\
\hline 44.85 & 0.78 & $13 \%$ & 117.76 & 0.89 & $57 \%$ & 165.78 & 0.89 & $47 \%$ \\
\hline 49.73 & 1.38 & $19 \%$ & 120.91 & 1.00 & $68 \%$ & 173.46 & 0.99 & $58 \%$ \\
\hline 59.38 & 1.25 & $21 \%$ & 124.90 & 1.09 & $68 \%$ & 177.56 & 0.97 & $101 \%$ \\
\hline 63.90 & 1.22 & $17 \%$ & 128.87 & 0.86 & $38 \%$ & 180.29 & 1.22 & $125 \%$ \\
\hline 77.27 & 0.81 & $11 \%$ & 136.73 & 0.87 & $37 \%$ & 184.58 & 1.00 & $92 \%$ \\
\hline 86.46 & 1.04 & $22 \%$ & 141.62 & 0.72 & $47 \%$ & 188.35 & 1.10 & $114 \%$ \\
\hline 93.23 & 0.86 & $43 \%$ & 145.41 & 0.70 & $48 \%$ & 191.84 & 1.63 & $302 \%$ \\
\hline 93.91 & 0.91 & $33 \%$ & 150.17 & 0.83 & $75 \%$ & 192.50 & 0.96 & $121 \%$ \\
\hline 103.62 & 1.01 & $33 \%$ & 152.07 & 0.75 & $86 \%$ & 197.92 & 1.21 & $89 \%$ \\
\hline
\end{tabular}

(a)

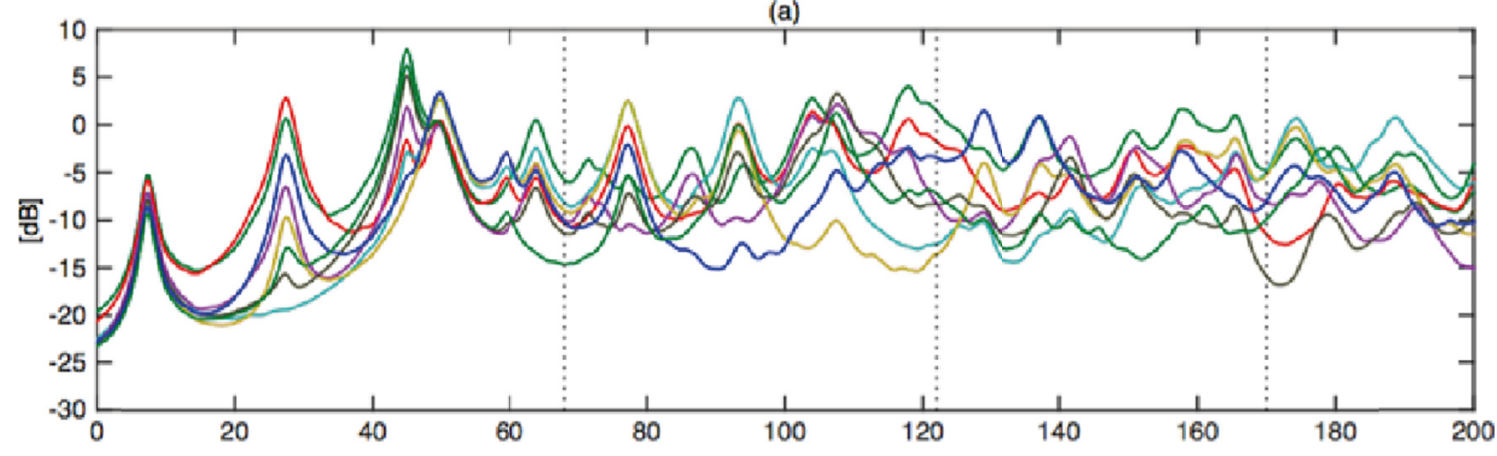

(b)

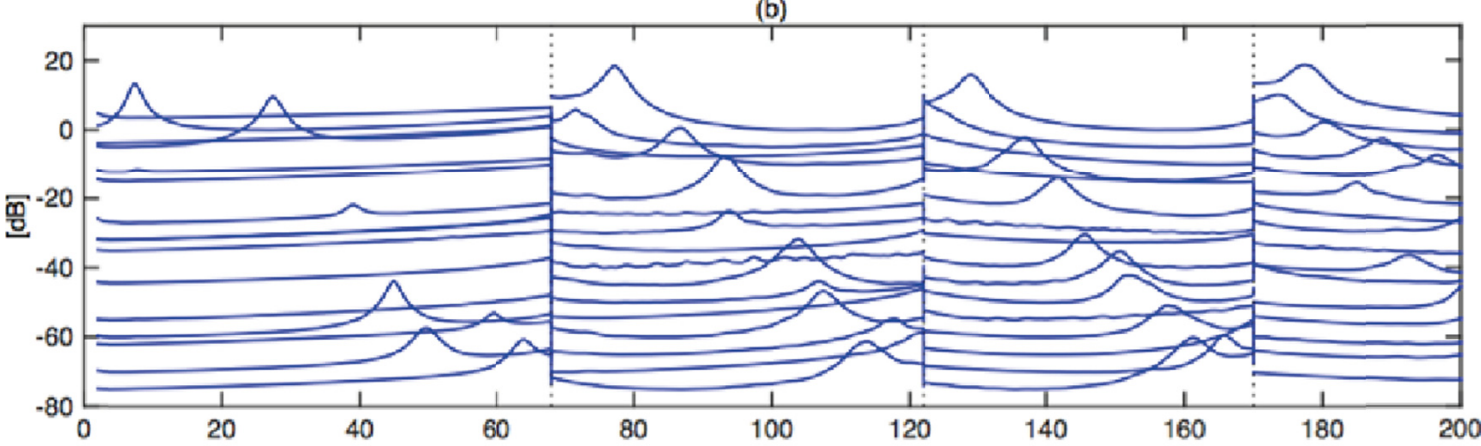

(c)

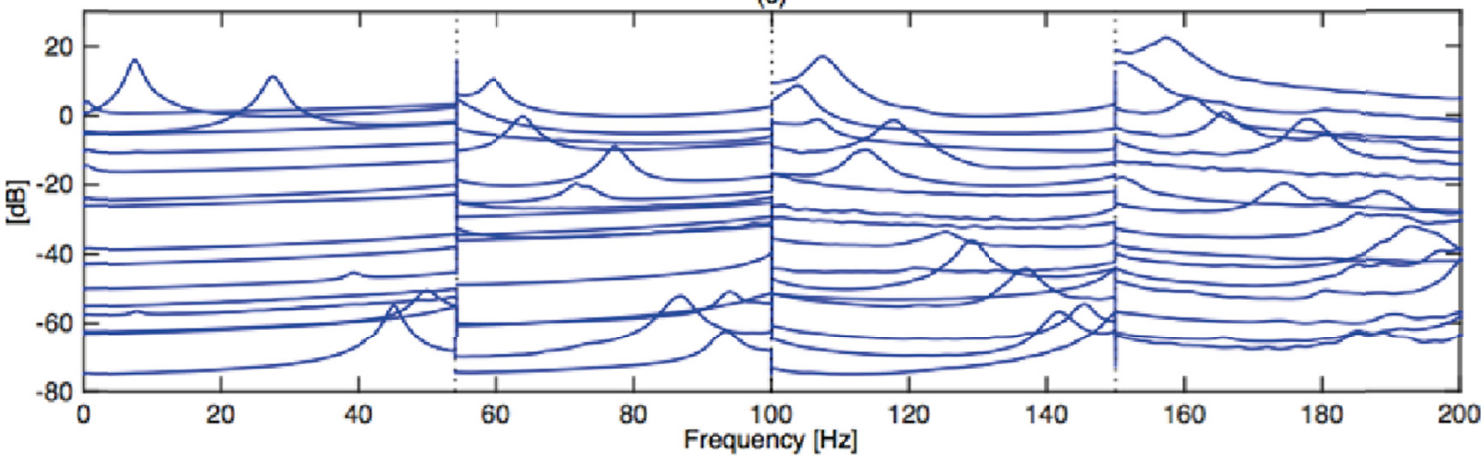

Fig. 12. Power spectral densities of a) output measurements and of b-c) separated modal responses with two different but complimentary configurations based on four subbands ( $M=8$ channels with data augmentation). 
Table 13

Identified modal parameters together with the estimated modal overlap factors ( $M=8$ channels with data augmentation).

\begin{tabular}{|c|c|c|c|c|c|c|c|c|}
\hline Freq. (Hz) & Damp. (\%) & MOF & Freq. (Hz) & Damp. (\%) & MOF & Freq. (Hz) & Damp. (\%) & MOF \\
\hline 7.31 & 0.93 & $1 \%$ & 106.78 & 1.34 & $144 \%$ & 156.19 & 0.28 & $32 \%$ \\
\hline 26.96 & 1.38 & $5 \%$ & 107.46 & 1.02 & $63 \%$ & 157.93 & 0.87 & $109 \%$ \\
\hline 38.90 & 1.82 & $16 \%$ & 113.71 & 1.13 & $53 \%$ & 161.24 & 0.87 & $75 \%$ \\
\hline 44.83 & 0.69 & $12 \%$ & 117.11 & 1.18 & $92 \%$ & 165.45 & 1.17 & $53 \%$ \\
\hline 49.43 & 1.35 & $18 \%$ & 119.69 & 0.33 & $21 \%$ & 175.94 & 0.93 & $58 \%$ \\
\hline 59.71 & 1.21 & $20 \%$ & 124.67 & 1.72 & $97 \%$ & 176.63 & 0.45 & $75 \%$ \\
\hline 63.69 & 1.19 & $18 \%$ & 128.49 & 0.73 & $32 \%$ & 180.11 & 1.15 & $108 \%$ \\
\hline 76.88 & 0.79 & $11 \%$ & 136.52 & 0.79 & $33 \%$ & 184.28 & 1.06 & $99 \%$ \\
\hline 86.46 & 1.02 & $22 \%$ & 141.61 & 0.75 & $47 \%$ & 187.97 & 0.96 & $92 \%$ \\
\hline 93.17 & 0.81 & $40 \%$ & 145.53 & 0.68 & $44 \%$ & 192.16 & 0.86 & $124 \%$ \\
\hline 94.11 & 1.12 & $41 \%$ & 150.47 & 0.75 & $65 \%$ & 193.28 & 1.03 & $242 \%$ \\
\hline 103.47 & 0.94 & $31 \%$ & 152.50 & 0.67 & $71 \%$ & 195.46 & 0.94 & $168 \%$ \\
\hline
\end{tabular}

mode 1

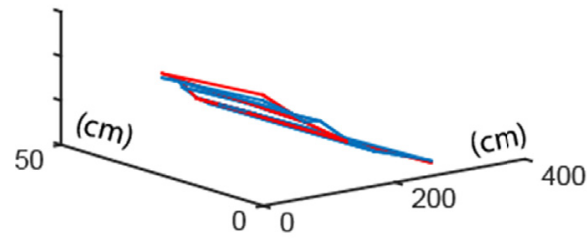

mode 3

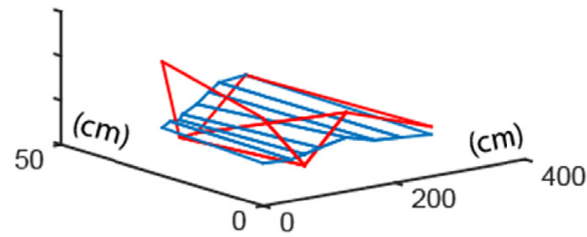

mode 5

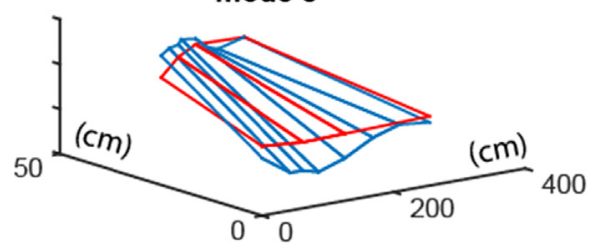

mode 2

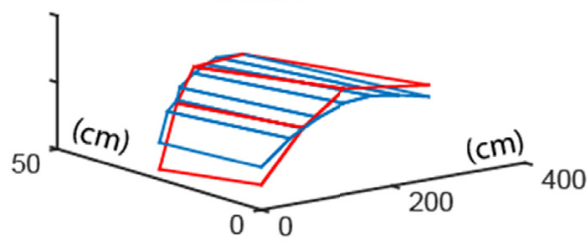

mode 4

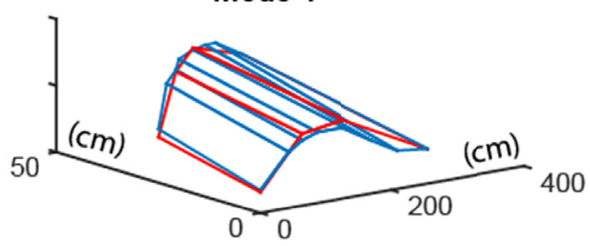

mode 6

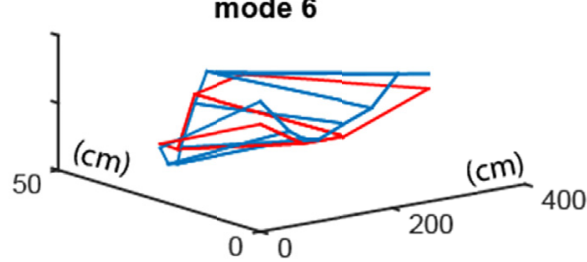

Fig. 13. First sixth reconstructed (unscaled) mode shapes of the wind turbine blade (deflection in vertical direction) from $M=16$ channels (blue) and $M=8$ channels (red). (For interpretation of the references to colour in this figure legend, the reader is referred to the web version of this article.)

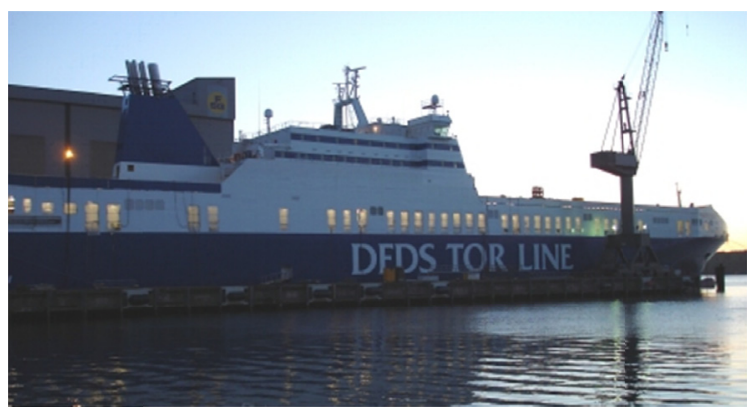

Fig. 14. Roll-on roll-off ship structure. 
(a)

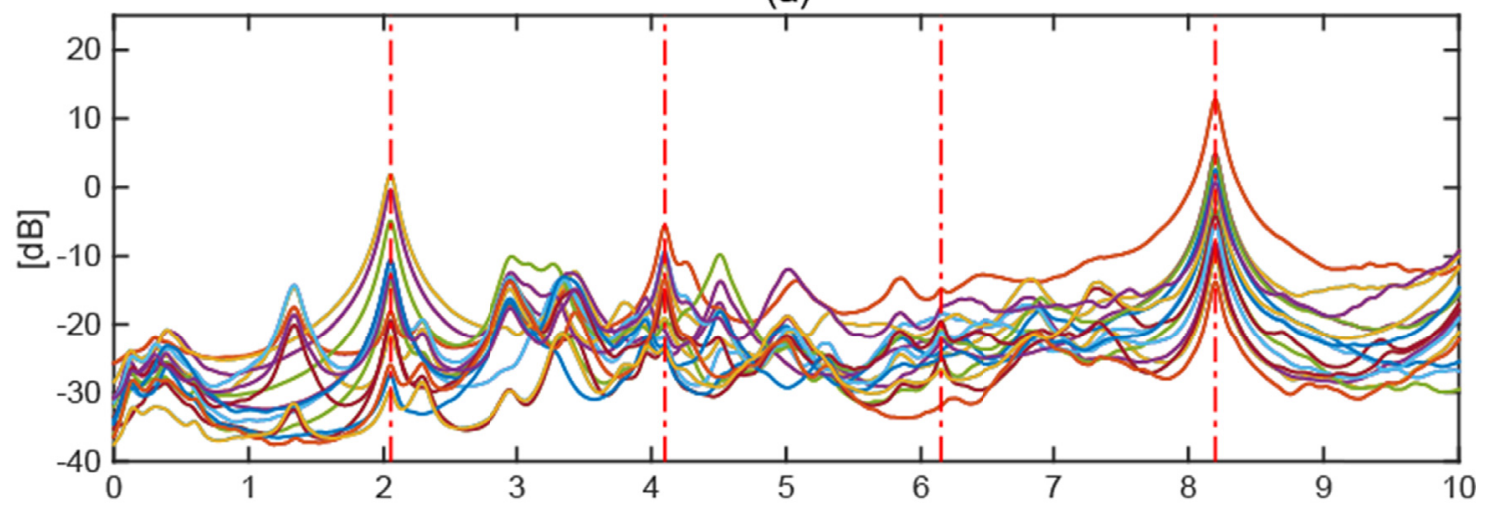

(b)

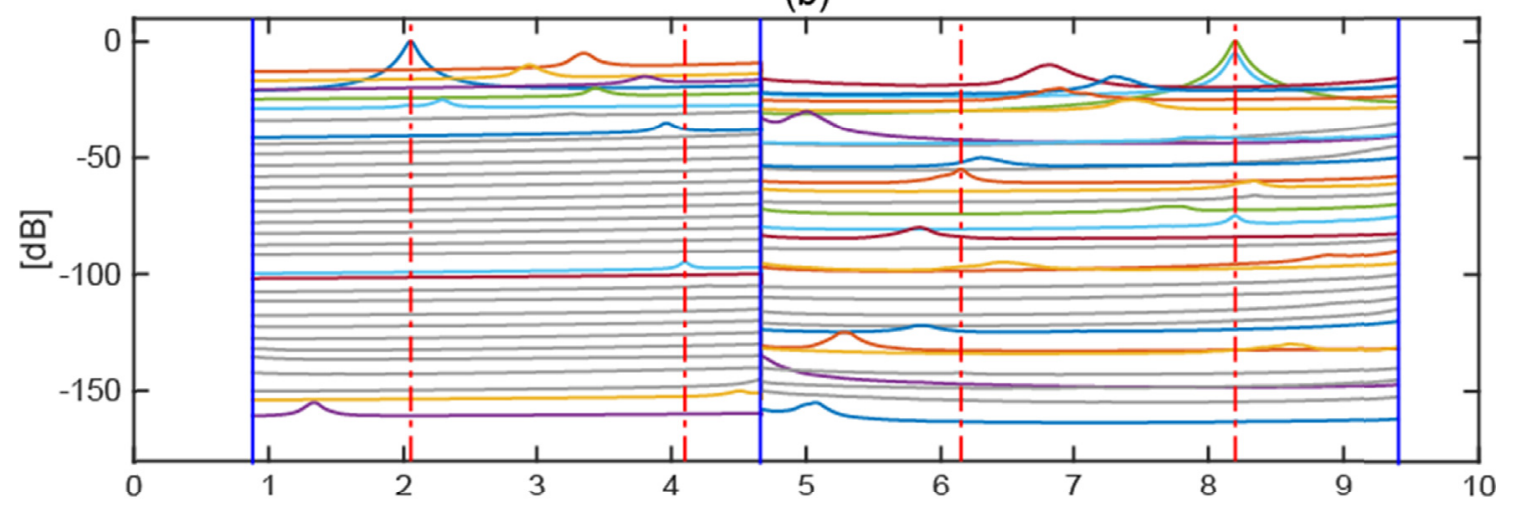

(c)

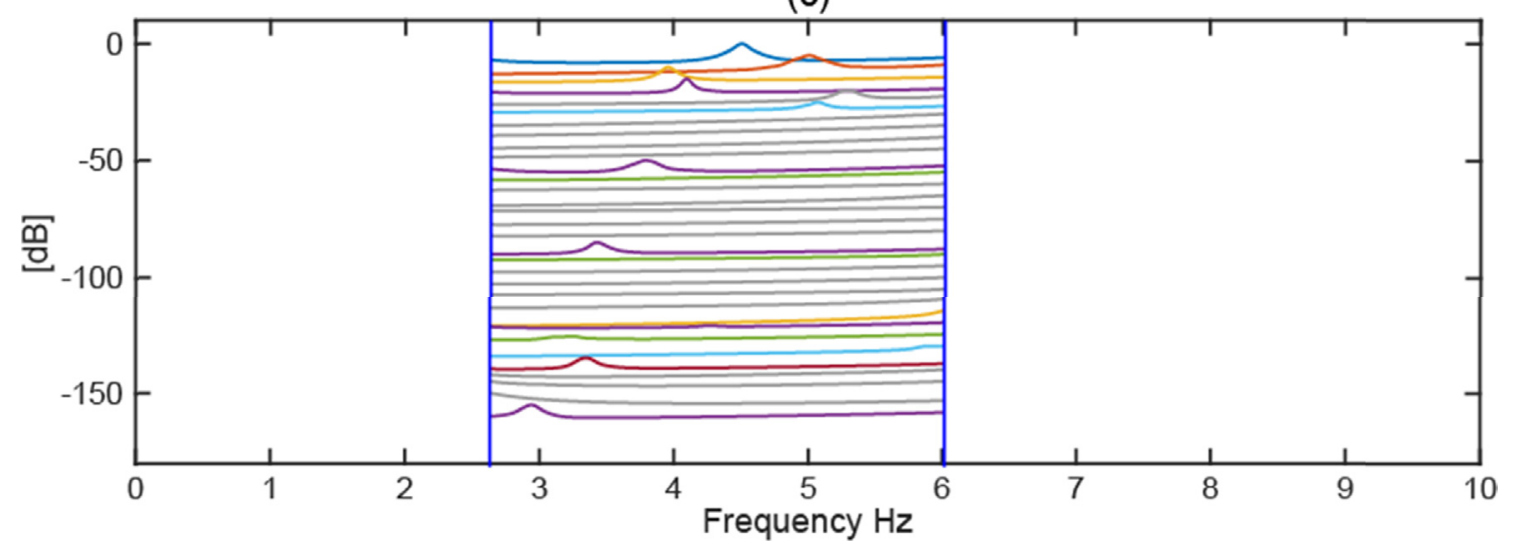

Fig. 15. Power spectral densities of a) output measurements of the roll-on roll-off ship and of b) separated modal responses with a DA factor of 2 in bands [0.88; 4.67] $\mathrm{Hz}$ and $[4.67 ; 9.4] \mathrm{Hz}$ and $\mathrm{c}$ ) in band [2.64; 6.01] Hz. Red dotted vertical lines indicate the locations of the engine harmonics. (For interpretation of the references to colour in this figure legend, the reader is referred to the web version of this article.)

deformations can hardly be assessed by only 8 sampling points on the blade, the flexural deformations match very well in the two configurations.

\subsubsection{Ship data}

The last set of data comes from a roll-on roll-off ship testing [35] (see Fig. 14). Data are collected for a period of 90 min with sampling frequency $F_{S}=128 \mathrm{~Hz}$. The frequency range of interest is [0;10] Hz. The ship engine is operating at $123 \mathrm{rpm}$ and it has a four bladed propeller with controllable pitch. Sixteen measurement channels are provided. 
Table 14

Identified engine harmonics (natural frequencies and damping ratios).

\begin{tabular}{ll}
\hline Frequency $(\mathrm{Hz})$ & Damping ratio (\%) \\
\hline 2.04 & $0+0.64 \mathrm{i}$ \\
4.15 & $0+1.30 \mathrm{i}$ \\
6.09 & $0+0.44 \mathrm{i}$ \\
8.20 & $0+0.04 \mathrm{i}$ \\
\hline
\end{tabular}

Table 15

Identified modal parameters (natural frequencies and damping ratios) and modal overlap factors.

\begin{tabular}{|c|c|c|c|c|c|}
\hline Freq. $(\mathrm{Hz})$ & Damp. (\%) & MOF & Freq. (Hz) & Damp. (\%) & MOF \\
\hline 1,32 & 1,51 & $4 \%$ & 5,27 & 1,97 & $64 \%$ \\
\hline 2,26 & 0,81 & $5 \%$ & 5,70 & 2,94 & $119 \%$ \\
\hline 2,94 & 1,04 & $12 \%$ & 5,83 & 1,63 & $62 \%$ \\
\hline 3,25 & 2,34 & $60 \%$ & 6,31 & 1,49 & $62 \%$ \\
\hline 3,45 & 0,56 & $14 \%$ & 6,44 & 2,76 & $143 \%$ \\
\hline 3,80 & 1,29 & $38 \%$ & 6,81 & 1,21 & $37 \%$ \\
\hline 3,96 & 0,65 & $15 \%$ & 7,32 & 1,09 & $52 \%$ \\
\hline 4,50 & 0,80 & $14 \%$ & 7,42 & 1,09 & $76 \%$ \\
\hline 4,98 & 1,41 & $51 \%$ & 7,74 & 1,60 & $89 \%$ \\
\hline 5,05 & 0,54 & $38 \%$ & 7,98 & 1,20 & $49 \%$ \\
\hline
\end{tabular}

This case is challenging in two aspects. First, the PSDs of the operational responses evidence a high modal overlap, to an extent where the modal behavior of the structure is hardly discernible (see Fig. 15(a)). Next, the presence of harmonics due to the engine operation is expected and may interfere with OMA.

Since many more modes are expected than the available number of channels, the frequency axis is divided in 3 bands, $[0.88 ; 4.67] \mathrm{Hz},[4.67 ; 9.4] \mathrm{Hz}$ and $[2.64 ; 6.01] \mathrm{Hz}$, where the last band purposely intersects with the first two in order to resolve modes at their frequency interface at $4.67 \mathrm{~Hz}$. In addition, a DA factor of $2\left(A_{i}=1, i=1,2,3\right)$ is systematically used with a one-sample shift $(p=1)$, thus resulting in data of dimension 32. The results of the separation are displayed in Fig. 15(b-c), where it is seen that all modes have been well resolved. Although the engine harmonics are separated together with the structural modes, they can be easily recognized because the identification procedure assigns them with inconsistent damping ratios that come out being complex with zero real parts, as reported in Table 14 . The values of the identified modal parameters are all listed in Table 15. It is again checked that separation can be effectively performed even in the presence of high modal overlap, with MOFs actually greater than $100 \%$.

\section{Conclusions}

This paper proposes a comprehensive methodology for the blind separation and identification of modes in the context of operational modal analysis. It is especially dedicated to largely underdetermined scenarios and therefore fixes a persisting limitation of current blind source separation algorithms. The approach takes advantage of the reformulation of second-order BSS in the frequency domain for decomposing a large underdetermined problem into several (over)determined problems in subbands. A strategy of data augmentation has also been introduced to deal with severely underdetermined scenarios and a new identification method has been devised to estimate the modal parameters of the structural modes after being separated in subbands. The method turns out user-friendly since the only intervention of the end-user is the selection of frequency bands, a rather intuitive process which can be achieved by "dragging the mouse". We believe that this is no more demanding than state-of-the-art solutions based on SSI, which require the inspection of a stabilization diagram to manually select the estimated modes. The proposed methodology has been purposely illustrated on several numerical and experimental data in order to demonstrate its practical feasibility; very satisfying results have been obtained even in difficult situations with complex modes, high modal overlap, singular modes, presence of engine harmonics and limited number of channels. One possible perspective is to refine the data augmentation strategy in order to reduce the approximation errors introduced in the mass and stiffness lines.

\section{Acknowledgments}

This work was supported by the Labex CeLyA (Centre Lyonnais d'Acoustique) of University of Lyon (ANR-10-LABX-0060.

\section{References}

[1] P. Van Overschee, B. De Moor, W. Dehandschutter, J. Swevers, A subspace algorithm for the identification of discrete time frequency domain power spectra, Automatica 33 (12) (1997) 2147-2157. 
[2] E. Reynders, System identification methods for (operational) modal analysis: review and comparison, Archives Comput. Methods Eng. 19 (1) (2012) $51-124$.

[3] M.H. Masjedian, M. Keshmiri, A review on operational modal analysis researches: classification of methods and applications, IOMAC 2009-3rd Int. Oper. Modal Anal. Conf. (2009) 707-716.

[4] A. Cichocki, S. Amari, Adaptive Blind Signal and Image Processing: Learning Algorithms and Applications, first ed., Wiley, 2002.

[5] J. Antoni, S. Chauhan, Second order blind source separation techniques (SO-BSS) and their relation to stochastic subspace identification (SSI) algorithm, in: Proceedings of the 28th IMAC, Jacksonville, FL, USA, 2010.

[6] A. Sadhu, S. Narasimhan, J. Antoni, A review of output-only structural mode identification literature employing blind source separation methods, Mech. Syst. Signal Process. 94 (2017) 415-431.

[7] G. Kerschen, F. Poncelet, J.-C. Golinval, Physical interpretation of independent component analysis in structural dynamics, Mech. Syst. Signal Process. 21 (2007) 1561-1575.

[8] F. Poncelet, G. Kerschen, J.-C. Golinval, Experimental modal analysis using blind source separation techniques, in: Proceedings of ISMA International Conference on Noise and Vibration Engineering, Katholieke Universiteit Leuven, Belgium, 2006.

[9] F. Poncelet, G. Kerschen, J.-C. Golinval, D. Verhelst, Output-only modal analysis using blind source separation techniques, Mech. Syst. signal Process. 21 (6) (2007) 2335-2358.

[10] S. Chauhan, R. Martell, R.J. Allemang, D.L. Brown, Application of independent component analysis and blind source separation techniques to operational modal analysis, in: Proceedings of the 25th IMAC, Orlando, FL, USA, 2007.

[11] S.I. McNiell, D.C. Zimmerman, A framework for blind modal identification using joint approximate diagonalization, Mech. Syst. Signal Process. 22 (2008) 1526-1548.

[12] J. Antoni, S. Chauhan, A study and extension of second-order blind source separation to operational modal analysis, J. Sound Vib. 332 (2013) 1079-1106.

[13] Y. Yang, S. Nagarajaiah, Blind modal identification of output-only structures in time-domain based on complexity pursuit, Earthq. Eng. Struct. Dyn. 42 (13) (2013) 1885-1905.

[14] Y. Yang, S. Nagarajaiah, Output-only modal identification by compressed sensing: non-uniform low-rate random sampling, Mech. Syst. Signal Process. 56-57 (2015) 15-34.

[15] Y. Yang, S. Nagarajaiah, Structural damage identification via a combination of blind feature extraction and sparse representation classification, Mech. Syst. Signal Process. 45 (2014) 1-23.

[16] J. Antoni, R. Castiglione, L. Garibaldi, Interpretation and generalization of complex pursuit for the blind separation of modal contributions, Mech. Syst. Signal Process. 85 (2017) 773-788.

[17] A. Smilde, R. Bro, P. Geladi, Multi-way Analysis with Applications in the Chemical Sciences, first ed., John Wiley and Sons, 2004.

[18] J.D. Carroll, J.J. Chang, Analysis of individual differences in multidimensional scaling via an n-way generalization of Eckart-Young decomposition, Psychometrika 35 (1970) 283-319.

[19] R.A. Harshman, Foundations of the PARAFAC procedure: models and conditions for an explanatory multi-model factor analysis, UCLA Work. Pap. Phonetics $16(1)(1970) 84$.

[20] J. Antoni, S. Chauhan, An alternating least squares (ALS) based blind source separation algorithm for operational modal analysis, in: Proceedings of the 29th IMAC, Jacksonville, FL, USA, 2011.

[21] A. Sadhu, B. Hazra, S. Narasimhan, Decentralized modal identification of structures using parallel factor decomposition and sparse blind source separation, Mech. Syst. Signal Process. 41 (1-2) (2013) 396-419.

[22] A. Sadhu, B. Hazra, S. Narasimhan, Ambient modal identification of structures equipped with tuned mass dampers using parallel factor blind source separation, Smart Struct. Syst. 13 (2) (2014) 257-280.

[23] A. Sadhu, A. Goldack, S. Narasimhan, Ambient modal identification using multi-rank parallel factor decomposition, Struct. Control Health Monit. 22 (4) (2015) 595-614.

[24] M. Zibulevsky, B. Pearlmutter, Blind source separation by sparse decomposition, Neural Comput. 13 (4) (2001) 863-883.

[25] A. Aissa El Bey, N. Linh-Trung, K. Abed-Meraim, A. Belouchrani, Y. Grenier, Underdetermined blind separation of non-disjoint sources in the timefrequency domain, IEEE Trans. Signal Process. 55 (3) (2007) 897-907.

[26] P. Kisilev, M. Zibulevsky, Y. Yehoshua, A multiscale framework for blind separation of linearly mixed signals, J. Mach. Learn. Res. 4 (2003) 1339-1365.

[27] F. Abrard, Y. Deville, A time-frequency blind signal separation method applicable to underdetermined mixtures of dependent sources, Signal Process. 85 (2005) 1389-1403.

[28] M.V. Hulle, Clustering approach to square and non-square blind source separation, in: IEEE Workshop on Neural Networks for Signal Processing, 1999, pp. $315-323$.

[29] B. Swaminathan, B. Sharma, S. Chauhan, Utilization of blind source separation techniques for modal analysis, structural dynamics 3, in: Part of the Series Conference Proceedings of the Society for Experimental Mechanics Series, 2011, pp. 189-206.

[30] K. Yu, K. Yang, Y. Bai, Estimation of modal parameters using the sparse component analysis based underdetermined blind source separation, Mech. Syst. Signal Process. 45 (2014) 302-316.

[31] S. Qin, J. Guo, C. Zhu, Sparse component analysis using time-frequency representations for operational modal analysis, Sensors 15 (3) (2015) 6497-6519.

[32] Y. Jin, S. Qin, J. Guo, C. Zhu, Output-only modal identification based on hierarchical Hough transform, J. Mech. Sci. Technol. 30 (7) (2016) 2941-2951.

[33] S.F. Ghahari, M.A. Ghannad, E.T. Taciroglu, Blind identification of soil-structure systems, Soil Dyn. Earthq. Eng. 45 (2013) $56-69$.

[34] J. Antoni, S. Chauhan, M. Monnier, K. Gryllias, Least action criteria for blind separation of structural modes, Mech. Industry 14 (6) (2014) 397-411.

[35] http://www.me.mtu.edu/imac_oma/(accessed 10/05/2016).

[37] S. Degerine, E. Kane, A comparative study of approximate joint diagonalization algorithms for blind source separation in presence of additive noise, signal processing, IEEE Trans. 55 (6) (2007) 3022-3031.

[38] T. Katayama, Subspace Methods for System Identification, first ed., Springer, 2005.

[39] J.-N. Juang, Applied System Identification, first ed., Prentice Hall, New Jersey, 1994. 